Pacific

Journal of

Mathematics

\title{
CONFORMALLY OSSERMAN MANIFOLDS
}

YURI NIKOLAYEVSKY 


\title{
CONFORMALLY OSSERMAN MANIFOLDS
}

\author{
YURI NIKOLAYEVSKY
}

To the memory of Novica Blažić (1959-2005), a remarkable mathematician and a wonderful person.

\begin{abstract}
An algebraic curvature tensor is called Osserman if the eigenvalues of the associated Jacobi operator are constant on the unit sphere. A Riemannian manifold is called conformally Osserman if its Weyl conformal curvature tensor at every point is Osserman. We prove that a conformally Osserman manifold of dimension $n \neq 3,4,16$ is locally conformally equivalent either to a Euclidean space or to a rank-one symmetric space.
\end{abstract}

\section{Introduction}

An algebraic curvature tensor $\mathscr{R}$ on a Euclidean space $\mathbb{R}^{n}$ is a $(3,1)$ tensor having the same symmetries as the curvature tensor of a Riemannian manifold. For $X \in \mathbb{R}^{n}$, the Jacobi operator $\mathscr{R}_{X}: \mathbb{R}^{n} \rightarrow \mathbb{R}^{n}$ is defined by $\mathscr{R}_{X} Y=\mathscr{R}(X, Y) X$. The Jacobi operator is symmetric, and $\mathscr{R}_{X} X=0$ for all $X \in \mathbb{R}^{n}$.

Definition 1.1. An algebraic curvature tensor $\mathscr{R}$ is Osserman if the eigenvalues of the Jacobi operator $\mathscr{R}_{X}$ do not depend on the choice of a unit vector $X \in \mathbb{R}^{n}$.

One of the algebraic curvature tensors naturally associated to a Riemannian manifold (apart from the curvature tensor itself) is the Weyl conformal curvature tensor.

Definition 1.2. A Riemannian manifold is (pointwise) Osserman if its curvature tensor at every point is Osserman. It is conformally Osserman if its Weyl tensor everywhere at every point is Osserman.

It is well known (and easy to check directly) that a Riemannian space locally isometric to a Euclidean space or to a rank-one symmetric space is Osserman. The question of whether the converse is true ("every pointwise Osserman manifold is flat or locally rank-one symmetric") is known as the Osserman conjecture [1990]. The first result on the Osserman conjecture, the affirmative answer for manifolds of

MSC2000: 53B20, 53A30.

Keywords: Osserman manifold, Weyl tensor, Jacobi operator, Clifford structure.

Supported by the FSTE grant. 
dimension not divisible by 4, was published before the conjecture itself [Chi 1988]. In the following two decades, substantial progress was made in understanding Osserman and related classes of manifolds, both in the Riemannian and pseudoRiemannian settings; see the books [Gilkey 2001; 2007; García-Río et al. 2002].

The Osserman conjecture is proved in the most cases, exception being when the dimension of an Osserman manifold is 16 and one of the eigenvalues of the Jacobi operator has multiplicity 7 or 8 [N 2003; 2004; 2005; 2006]. The main difficulty in proving the conjecture in these remaining cases lies in the fact that the Cayley projective plane (and its hyperbolic dual) are Osserman, with the multiplicities of the nonzero eigenvalues of the Jacobi operator being exactly 7 and 8; moreover, the curvature tensor of the Cayley projective plane is essentially different from that of the other rank-one symmetric spaces, as it does not admit a Clifford structure (see Section 2 for details). This is the only known Osserman curvature tensor without a Clifford structure, and to prove the Osserman conjecture in full, it would be very desirable to show that there are no other exceptions.

The study of conformally Osserman manifolds was started by Blažić and Gilkey [2004] and was continued in [Blažić and Gilkey 2005; Blažić et al. 2005; Gilkey 2007; Blažić et al. 2008]. Every Osserman manifold is conformally Osserman (which easily follows from the formula for the Weyl tensor and the fact that every Osserman manifold is Einstein), since also every manifold is locally conformally equivalent to an Osserman manifold.

Theorem 1.3 (main result). A connected $C^{\infty}$ Riemannian conformally Osserman manifold of dimension $n \neq 3,4,16$ is locally conformally equivalent to a Euclidean space or to a rank-one symmetric space.

This theorem answers the conjecture made in [Blažić et al. 2005], with three exceptions. (For conformally Osserman manifolds of dimension $n>6$, not divisible by 4 , this conjecture is proved in [Blažić and Gilkey 2004, Theorem 1.4].)

Note that the nature of the three excepted dimensions in Theorem 1.3 is different. In dimension three, the Weyl tensor vanishes, hence giving no information about the manifold at all. In dimension four, even a "genuine" pointwise Osserman manifold may not be locally symmetric (see the examples of "generalized complex space forms" in [Gilkey et al. 1995, Corollary 2.7] and [Olszak 1989]). As proved in [Chi 1988], the Osserman conjecture is still true in dimension four, but in a more restrictive version: One requires the eigenvalues of the Jacobi operator to be constant on the whole unit tangent bundle (a Riemannian manifold having this property is called globally Osserman). One might wonder whether the conformal counterpart of this result is true. Blažić and Gilkey [2005] found the elegant characterization that a four-dimensional Riemannian manifold is conformally Osserman if and only if it is either self-dual or anti-self-dual. 
In dimension 16, both the conformal and the original Osserman conjecture remain open; for partial results, see [N 2005; 2006] in the Riemannian case and Theorem 3.1 in the conformal case.

As a rather particular case of Theorem 1.3, we obtain an analogue of the WeylSchouten theorem for rank-one symmetric spaces: A Riemannian manifold of dimension greater than four having "the same" Weyl tensor as that of one of the complex/quaternionic projective spaces or their noncompact duals is locally conformally equivalent to that space. More precisely:

Theorem 1.4. Let $M_{0}^{n}$ denote one of the spaces $\mathbb{C} P^{n / 2}, \mathbb{C} H^{n / 2}, \mathbb{M} P^{n / 4}$ or $\mathbb{H} H^{n / 4}$, and let $W_{0}$ be the Weyl tensor of $M_{0}^{n}$ at some point $x_{0} \in M_{0}^{n}$. Suppose that for every point $x$ of a Riemannian manifold $M^{n}$ with $n>4$ there exists a linear isometry $\imath: T_{x} M^{n} \rightarrow T_{x_{0}} M_{0}^{n}$ that maps the Weyl tensor of $M^{n}$ at $x$ on a positive multiple of $W_{0}$. Then $M^{n}$ is locally conformally equivalent to $M_{0}^{n}$.

The claim follows from [Blažić and Gilkey 2004, Theorem 1.4] for $M_{0}^{n}=\mathbb{C} P^{n / 2}$, $\mathbb{C} H^{n / 2}$ and $n>6$. The fact that the dimension $n=16$ is not excluded (as compared to Theorem 1.3) follows from Theorem 3.1.

We assume all the object (manifolds, metrics, vector and tensor fields) to be smooth (of class $C^{\infty}$ ), although all the results remain valid for class $C^{k}$, with sufficiently large $k$.

The paper is organized as follows. In Section 2, we give some background on Osserman algebraic curvature tensors and on Clifford structures and prove some technical lemmas. The proof of Theorem 1.3 is given in Section 3. Theorem 1.3 is deduced from a more general Theorem 3.1. We first prove the local version using the second Bianchi identity, and then the global version by showing that the "algebraic type" of the Weyl tensor is the same at all points of a connected conformally Osserman Riemannian manifold (in particular, a nonzero Osserman Weyl tensor cannot degenerate to zero).

\section{Algebraic curvature tensors with a Clifford structure}

2.1. Clifford structure. The requirement that an algebraic curvature tensor $\mathscr{R}$ be Osserman is algebraically quite restrictive. In most cases, such a tensor can be obtained by the following construction, suggested in [Gilkey et al. 1995], which generalizes the curvature tensor of complex and quaternionic projective space.

Definition 2.2. A Clifford structure $\operatorname{Cliff}\left(v ; J_{1}, \ldots, J_{v} ; \lambda_{0}, \eta_{1}, \ldots, \eta_{v}\right)$ on $\mathbb{R}^{n}$ is a set of $v \geq 0$ anticommuting almost Hermitian structures $J_{i}$ and $v+1$ real numbers $\lambda_{0}, \eta_{1}, \ldots \eta_{v}$, with $\eta_{i} \neq 0$. An algebraic curvature tensor $\mathscr{R}$ on $\mathbb{R}^{n}$ has a Clifford 
structure $\operatorname{Cliff}\left(v ; J_{1}, \ldots, J_{v} ; \lambda_{0}, \eta_{1}, \ldots, \eta_{v}\right)$ if

$$
\begin{aligned}
\mathscr{R}(X, Y) Z=\lambda_{0}(\langle X, Z\rangle Y-\langle Y, Z\rangle X) & \\
& +\sum_{i=1}^{\nu} \eta_{i}\left(2\left\langle J_{i} X, Y\right\rangle J_{i} Z+\left\langle J_{i} Z, Y\right\rangle J_{i} X-\left\langle J_{i} Z, X\right\rangle J_{i} Y\right) .
\end{aligned}
$$

When it does not create ambiguity, we write $\operatorname{Cliff}\left(v ; J_{1}, \ldots, J_{v} ; \lambda_{0}, \eta_{1}, \ldots, \eta_{v}\right)$ simply as Cliff(v).

Remark 2.3. Definition 2.2 implies that the operators $J_{i}$ are skew-symmetric and orthogonal and satisfy the equations

$$
\left\langle J_{i} X, J_{j} X\right\rangle=\delta_{i j}\|X\|^{2} \quad \text { and } \quad J_{i} J_{j}+J_{j} J_{i}=-2 \delta_{i j} \text { id }
$$

for all $i, j=1, \ldots, v$ and all $X \in \mathbb{R}^{n}$. This implies that every algebraic curvature tensor with a Clifford structure is Osserman, as by (2-1) the Jacobi operator has the form $\mathscr{R}_{X} Y=\lambda_{0}\left(\|X\|^{2} Y-\langle Y, X\rangle X\right)+\sum_{i=1}^{v} 3 \eta_{i}\left\langle J_{i} X, Y\right\rangle J_{i} X$. So for a unit vector $X$, the eigenvalues of $\mathscr{T}_{X}$ are $\lambda_{0}$ (of multiplicity $n-1-v$ if $v<n-1$ ), 0 , and $\lambda_{0}+3 \eta_{i}$ for $i=1, \ldots, v$.

The converse - every Osserman algebraic curvature tensor has a Clifford structure - is true in all dimensions but $n=16$ and also in many cases when $n=16$, as follows from [N 2005, Proposition 1 and the penultimate paragraph of the proof of Theorems 1 and 2], [N 2004, Proposition 1] and [N 2006, Proposition 2.1]. The only known counterexample is the curvature tensor $R^{\mathrm{OP}}$ of the Cayley projective plane (more precisely, any algebraic curvature tensor of the form $\mathscr{R}=a R^{\mathrm{O} P^{2}}+b R^{1}$, where $R^{1}$ is the curvature tensor of the unit sphere $S^{16}(1)$ and $a \neq 0$ ).

A Clifford structure Cliff $(v)$ on Euclidean $\mathbb{R}^{n}$ turns it into a Clifford module; see [Atiyah et al. 1964, Part 1], [Husemoller 1975, Chapter 11], and [Lawson and Michelsohn 1989, Chapter 1] for standard facts on Clifford algebras and Clifford modules). A Clifford algebra $\mathrm{Cl}(v)$ on $v$ generators $x_{1}, \ldots, x_{v}$ is an associative unital algebra over $\mathbb{R}$ defined by the relations $x_{i} x_{j}+x_{j} x_{i}=-2 \delta_{i j}$. The homomorphism $\sigma: \mathrm{Cl}(v) \rightarrow \operatorname{End}\left(\mathbb{R}^{n}\right)$ of associative algebras defined on generators by $\sigma\left(x_{i}\right)=J_{i}$ and $\sigma(1)=\mathrm{id}$ is a representation of $\mathrm{Cl}(v)$ on $\mathbb{R}^{n}$. Since all the $J_{i}$ are orthogonal and skew-symmetric, $\sigma$ gives rise to an orthogonal multiplication defined as follows. In the Euclidean space $\mathbb{R}^{v}$, fix an orthonormal basis $e_{1}, \ldots, e_{\nu}$. For every $u=\sum_{i=1}^{v} u_{i} e_{i} \in \mathbb{R}^{v}$ and every $X \in \mathbb{R}^{n}$, define

$$
J_{u} X=\sum_{i=1}^{v} u_{i} J_{i} X
$$

(when $u=e_{i}$, we abbreviate $J_{e_{i}}$ to $J_{i}$ ). The map $J: \mathbb{R}^{v} \times \mathbb{R}^{n} \rightarrow \mathbb{R}^{n}$ defined by (2-2) is an orthogonal multiplication: $\left\|J_{u} X\right\|^{2}=\|u\|^{2}\|X\|^{2}$ (similarly, we can define an orthogonal multiplication $J: \mathbb{R}^{v+1} \times \mathbb{R}^{n} \rightarrow \mathbb{R}^{n}$ by $J_{u} X=u_{0} X+\sum_{i=1}^{v} u_{i} J_{i} X$ for $u=$ 
$\sum_{i=0}^{v} u_{i} e_{i} \in \mathbb{R}^{v+1}$, where $e_{0}, \ldots, e_{v}$ is an orthonormal basis for Euclidean $\mathbb{R}^{v+1}$ ). For $X \in \mathbb{R}^{n}$, denote

$$
\mathscr{g} X=\operatorname{Span}\left(J_{1} X, \ldots, J_{v} X\right) \text { and } \mathscr{I} X=\operatorname{Span}\left(X, J_{1} X, \ldots, J_{v} X\right) .
$$

We also use the complexified versions of these subspaces, which we denote by $\mathscr{\Phi}_{\mathbb{C}} X$ and $\Phi_{\mathbb{C}} X$ respectively for $X \in \mathbb{C}^{n}$.

If $\mathbb{R}^{n}$ is a $\mathrm{Cl}(v)$-module (equivalently, if there exists an algebraic curvature tensor with a Clifford structure $\operatorname{Cliff}(v)$ on $\left.\mathbb{R}^{n}\right)$, then

$$
v \leq 2^{b}+8 a-1, \quad \text { where } n=2^{4 a+b} c, c \text { is odd, and } 0 \leq b \leq 3 ;
$$

see, for instance, [Husemoller 1975, Theorem 11.8.2].

As a direct consequence of (2-3), we have the following inequalities.

Lemma 2.4. Let $\mathscr{R}$ be an algebraic curvature tensor with a Clifford structure $\operatorname{Cliff}(v)$ on $\mathbb{R}^{n}$. Suppose that $n>4$ and $n \neq 8,16$. Then

(i) $n \geq 3 v+3$, with equality only when $n=6$ and $v=1$, or $n=12$ and $v=3$, or $n=24$ and $v=7$

(ii) $n>4 v-2$, except when $n=24$ and $v=7$ or $n=32$ and $v=9$;

(iii) there exists an integer $l$ such that $v<2^{l}<n$.

Proof. Let $\rho(n)=2^{b}+8 a-1$, the right side of (2-3). Then $v \leq \rho(n)$. First suppose that $n=2^{m} c$, with $m=4 a+b \geq 6$, where $0 \leq b \leq 3$ and $c$ is odd. We claim that $n>4 \rho(n)$. Indeed, $n \geq 2^{m}=2^{4 a+b}$, so it suffices to show that $2^{4 a-2}>1+2^{3-b} a-2^{-b}$. The latter inequality follows from $2^{4 a-2}>1+8 a$, when $a \geq 2$, and is also true when $a=1$ and $b=2,3$. Since $n>4 \rho(n)$, (ii) is obvious, (i) is satisfied (since $\rho(n)>3$ ), and (iii) is satisfied with $l=m-1$.

In each of the remaining cases $\left(n=2^{m} c\right.$, with an odd $c$ and $\left.m=0, \ldots, 5\right)$, $\rho(n)$ can be computed explicitly and the claim follows by a routine check.

2.5. Clifford structures on $\mathbb{R}^{\mathbf{8}}$ and the octonions. The proof of Theorem 1.3 in the generic case uses that $v$ is small relative to $n$ (with the required estimates given in Lemma 2.4). However, in the case $n=8$, the number $v$ can be as large as 7 , according to (2-3). Consider this case in more detail. In [N 2004], it is shown that every Osserman algebraic curvature tensor $\mathscr{R}$ on $\mathbb{R}^{8}$ has a Clifford structure, and that either $\mathscr{R}$ has a Cliff(3) structure with $J_{1} J_{2}= \pm J_{3}$, or an existing $\operatorname{Cliff}(v)$ structure can be complemented to a Cliff(7) structure. More precisely:

Lemma 2.6. (1) Suppose $\mathscr{R}$ is an algebraic curvature tensor on $\mathbb{R}^{8}$ with Clifford structure $\operatorname{Cliff}\left(v ; J_{1}, \ldots, J_{v} ; \lambda_{0}, \eta_{1}, \ldots, \eta_{v}\right)$. Then exactly one of two possibilities may occur: either $\mathscr{R}$ has a Clifford structure Cliff(3) with $J_{1} J_{2}=J_{3}$, or there exist $7-v$ operators $J_{v+1}, \ldots, J_{7}$ such that $J_{1}, \ldots, J_{7}$ are anticommuting almost Hermitian structures with $J_{1} J_{2} \ldots J_{7}=\mathrm{id}_{\mathbb{R}^{8}}$ and $\mathscr{R}$ has a Clifford 
structure $\operatorname{Cliff}\left(7 ; J_{1}, \ldots, J_{7} ; \lambda_{0}-3 \xi, \eta_{1}+\xi, \ldots, \eta_{v}+\xi, \xi, \ldots, \xi\right)$ for any $\xi \neq-\eta_{i}, 0$.

(2) Let $\mathbb{O}$ be the octonion algebra with inner product defined by $\|u\|^{2}=u u^{*}$, where ${ }^{*}$ is the octonion conjugation, and let $\mathbb{O}^{\prime}=1^{\perp}$, the space of imaginary octonions. Then, in the second case in part (1), there exist linear isometries $\iota_{1}: \mathbb{R}^{8} \rightarrow \mathbb{O}$ and $\iota_{2}: \mathbb{R}^{7} \rightarrow \mathbb{O}^{\prime}$ such that the orthogonal multiplication (2-2) is given by $J_{u} X=\imath_{1}(X) \iota_{2}(u)$.

Proof. (1) This claim is proved in [N 2004, Lemma 5]. The proof is based on the fact that every representation $\sigma$ of $\mathrm{Cl}(v)$ on $\mathbb{R}^{8}$, except for the representations of $\mathrm{Cl}(3)$ with $J_{1} J_{2}= \pm J_{3}$, is a restriction of a representation of $\mathrm{Cl}(7)$ on $\mathbb{R}^{8}$ to $\mathrm{Cl}(v) \subset \mathrm{Cl}(7)$. It follows that the almost Hermitian structures $J_{1}, \ldots, J_{v}$ defined by $\sigma$ can be complemented by almost Hermitian structures $J_{v+1}, \ldots, J_{7}$ such that $J_{1}, \ldots, J_{7}$ anticommute, and so $\mathscr{R}$ can be written in the form (2-1), with a formal summation up to 7 on the right side (but with $\eta_{i}=0$ when $i=v+1, \ldots, 7$ ). To obtain a Cliff(7) structure for $\mathscr{R}$, according to Definition 2.2 , we only need to make all the $\eta_{i}$ nonzero. This can be done using the identity

$$
\langle X, Z\rangle Y-\langle Y, Z\rangle X=\sum_{i=1}^{7} \frac{1}{3}\left(2\left\langle J_{i} X, Y\right\rangle J_{i} Z+\left\langle J_{i} Z, Y\right\rangle J_{i} X-\left\langle J_{i} Z, X\right\rangle J_{i} Y\right)
$$

which is gotten from the polarized identity

$$
\|X\|^{2} Y-\langle X, Y\rangle X=\sum_{i=1}^{7}\left\langle J_{i} X, Y\right\rangle J_{i} X
$$

which is true because for $X \neq 0$ the vectors $\|X\|^{-1} X,\|X\|^{-1} J_{1} X, \ldots,\|X\|^{-1} J_{7} X$ form an orthonormal basis for $\mathbb{R}^{8}$. Then by (2-1), $\mathscr{R}$ has a Clifford structure $\operatorname{Cliff}\left(7 ; J_{1}, \ldots, J_{7} ; \lambda_{0}-3 \xi, \eta_{1}+\xi, \ldots, \eta_{v}+\xi, \xi, \ldots, \xi\right)$ for any $\xi \neq-\eta_{i}, 0$.

(2) This claim is proved in [N 2004, the beginning of Section 5.1]. The proof is based on the following. There are two nonisomorphic representations of $\mathrm{Cl}(7)$ on $\mathbb{R}^{8}$. By identifying $\mathbb{R}^{8}$ with the octonion algebra $\mathbb{O}$ via a linear isometry, these representations are given by the orthogonal multiplications $J_{u} X=u X$ and $J_{u} X=X u$ respectively [Lawson and Michelsohn 1989, Section I.8]. Since $(u X)^{*}=X^{*} u^{*}=$ $-X^{*} u$ for all $u, X \in \mathbb{O}$ with $u \perp 1$, the first representation is orthogonally equivalent to the second one, with the operators $J_{i}$ replaced by $-J_{i}$. Since changing the signs of the $J_{i}$ does not affect the form of the algebraic curvature tensor (2-1), we can always assume that a $\operatorname{Cliff}(7)$ structure for an algebraic curvature tensor on $\mathbb{R}^{8}$ is given by the orthogonal multiplication $J_{u} X=\iota_{1}(X) \iota_{2}(u)$.

In the proof of Theorem 1.3 for $n=8$, we will usually identify $\mathbb{R}^{8}$ with $\mathbb{O}$ and identify $\mathbb{R}^{7}$ with $\mathbb{O}^{\prime}$ via some fixed linear isometries $\iota_{1}$ and $\iota_{2}$, and we will simply 
write the orthogonal multiplication in the form

$$
J_{u} X=X u,
$$

where $X \in \mathbb{R}^{8}=\mathbb{O}$ and $u \in \mathbb{O}^{\prime}$. The proof of Theorem 1.3 for $n=8$ extensively uses computations in the octonion algebra $\mathbb{O}$, in particular, the standard identities

$$
\begin{aligned}
& a^{*}=2\langle a, 1\rangle 1-a, \quad\langle a, b\rangle=\left\langle a^{*}, b^{*}\right\rangle=\frac{1}{2}\left(a^{*} b+b^{*} a\right), \\
& a(a b)=a^{2} b, \quad\langle a, b c\rangle=\left\langle b^{*} a, c\right\rangle=\left\langle a c^{*}, b\right\rangle, \\
& \left(a b^{*}\right) c+\left(a c^{*}\right) b=2\langle b, c\rangle a, \quad\langle a b, a c\rangle=\langle b a, c a\rangle=\|a\|^{2}\langle b, c\rangle
\end{aligned}
$$

for any $a, b, c \in \mathbb{O}$, and the like; see for example [Harvey and Lawson 1982, Section IV]. It also uses the fact that $\mathbb{O}$ is a division algebra; in particular, any nonzero octonion is invertible: $a^{-1}=\|a\|^{-2} a^{*}$. We will also use the bioctonions $\mathbb{O} \otimes \mathbb{C}$, the algebra over the $\mathbb{C}$ that has same multiplication table as $\mathbb{O}$. Since all the identities above are polynomial, they still hold for bioctonions, with the complex inner product on $\mathbb{C}^{8}$, the underlying linear space of $\mathbb{O} \otimes \mathbb{C}$. However, the bioctonion algebra is not a division algebra (and has zero-divisors: $\left.\left(\mathrm{i} 1+e_{1}\right)\left(\mathrm{i} 1-e_{1}\right)=0\right)$.

The proof of Theorem 1.3 will require a technical lemma.

Lemma 2.7. (1) Let $J_{1}, \ldots, J_{v}$ be anticommuting almost Hermitian structures on $\mathbb{R}^{n}$, and let $F: \mathbb{R}^{n} \rightarrow \mathbb{R}^{n}$ be a homogeneous polynomial map of degree $m$ such that $F(X) \in \mathscr{S} X$ for all $X \in \mathbb{R}^{n}$. Suppose that $n>4$, and also $v \leq 3$ if $n=8$ and $v \leq 7$ if $n=16$. Then there exist homogeneous polynomials $c_{i}$ for $i=1, \ldots, v$ of degree $m-1$ such that $F(X)=\sum_{i=1}^{v} c_{i}(X) J_{i} X$.

With the same assumption, but with $\mathscr{I}$ replaced by $\mathscr{I}$, an additional homogeneous degree $m-1$ polynomial $c_{0}$ appears, and $c_{0}(X) X$ is added to $F(X)$.

(2) Let $J_{1}, \ldots, J_{v}$ be anticommuting almost Hermitian structures on $\mathbb{R}^{n}$. Suppose that $n>4$ and that $v \leq 3$ if $n=8$. Let $1 \leq k \leq v$ and let $a_{j}$ for $1 \leq j \leq v$ with $j \neq k$ be $v-1$ vectors in $\mathbb{R}^{n}$ such that

$$
\sum_{j \neq k}\left(\left\langle a_{j}, J_{k} Y\right\rangle J_{j} Y+\left\langle a_{j}, Y\right\rangle J_{k} J_{j} Y\right)=0 \quad \text { for all } Y \in \mathbb{R}^{n} .
$$

Then either $a_{j}=0$ for all $j \neq k$, or $v=1$, or $v=3, J_{1} J_{2}=\varepsilon J_{3}, \varepsilon= \pm 1$, and $a_{j}=J_{i} v$, where $\{i, j, k\}=\{1,2,3\}$ and $v \neq 0$.

(3) Let $N^{n}$ be a smooth Riemannian manifold and let $J_{1}, \ldots, J_{v}$ be anticommuting almost Hermitian structures on $N^{n}$. Suppose that for every nowhere vanishing smooth vector field $X$ on $N^{n}$, the distribution $\mathscr{E} X=\operatorname{Span}\left(J_{1} X, \ldots, J_{v} X\right)$ is smooth (that is, the $v$-form $J_{1} X \wedge \cdots \wedge J_{v} X$ is smooth). Then for every $x \in N^{n}$, there exists a neighborhood $U=U(x)$ and smooth anticommuting almost Hermitian structures $\tilde{J}_{1}, \ldots, \tilde{J}_{v}$ on $U$ such that $\operatorname{Span}\left(\tilde{J}_{1} X, \ldots, \tilde{J}_{v} X\right)=\operatorname{Span}\left(J_{1} X, \ldots, J_{v} X\right)$ for any vector field $X$ on $U$. 
Proof. (1) It is sufficient to prove the assertion for the case $F(X) \in \mathscr{I} X$.

Since for every $X \neq 0$, the vectors $X, J_{1} X, \ldots, J_{v} X$ are orthogonal and have the same length $\|X\|$, we have

$$
\|X\|^{2} F(X)=f_{0}(X) X+\sum_{i=1}^{v} f_{i}(X) J_{i} X,
$$

where $f_{0}(X)=\langle F(X), X\rangle$ and $f_{i}(X)=\left\langle F(X), J_{i} X\right\rangle$ are homogeneous polynomials of degree $m+1$ of $X$ (or possibly zeros). Taking the squared lengths of the both sides we get

$$
\|X\|^{2}\|F(X)\|^{2}=f_{0}^{2}(X)+\sum_{i=1}^{\nu} f_{i}^{2}(X),
$$

so the sum of squares of the $v+1$ polynomials $f_{0}(X), f_{1}(X), \ldots, f_{v}(X)$ is divisible by $\|X\|^{2}$. For $X=\left(x_{1}, \ldots, x_{n}\right)$, let $\left(\|X\|^{2}\right)$ be the ideal of $\mathbb{R}[X]$ generated by $\|X\|^{2}=\sum_{j} x_{j}^{2}$, and let $\mathbf{R}$ be the quotient of $\mathbb{R}[X]$ by this ideal. Let $\pi$ be the natural projection from $\mathbb{R}[X]$ to $\mathbf{R}$. We have $\sum_{i=0}^{v} \hat{f}_{i}^{2}=0$, where $\hat{f}_{i}=\pi f_{i}$. If at least one of the $\hat{f}_{i}$ is nonzero (say the $v$-th one), then $\sum_{i=0}^{v-1}\left(\hat{f}_{i} / \hat{f}_{v}\right)^{2}=-1$ in $\mathbb{F}$, the field of fractions of the ring $\mathbf{R}$. The field $\mathbb{E}$ is isomorphic to the field $\mathbb{L}_{n-1}=\mathbb{R}\left(x_{1}, \ldots, x_{n-1}, \sqrt{-d}\right)$, where $d=x_{1}^{2}+\cdots+x_{n-1}^{2}$ (an isomorphism from $\mathbb{L}_{n-1}$ to $\mathbb{F}$ is induced by the map $(a+b \sqrt{-d}) / c \rightarrow\left(a+b x_{n}\right) / c$, with $a, b, c \in$ $\mathbb{R}\left[x_{1}, \ldots, x_{n-1}\right]$ and $\left.c \neq 0\right)$. By [Pfister 1995, Theorem 3.1.4], the level of the field $\mathbb{L}_{n-1}$, the minimal number of elements whose sum of squares is -1 , is $2^{l}$, where $2^{l}<n \leq 2^{l+1}$. It follows that we arrive at a contradiction in all the cases when $v<2^{l}<n$. This means that $\hat{f}_{i}=0$ for all $i=0, \ldots, v$, so each of the $f_{i}$ is divisible by $\|X\|^{2}$ in $\mathbb{R}[X]$, so

$$
F(X)=\left(\|X\|^{-2} f_{0}(X)\right) X+\sum_{i=1}^{v}\left(\|X\|^{-2} f_{i}(X)\right) J_{i} X,
$$

with all the nonzero coefficients on the right side being homogeneous polynomials of degree $m-1$. The claim now follows from Lemma 2.4(iii).

(2) If $v=1$, Equation (2-6) is trivially satisfied. If $v=2$, the claim follows immediately by taking the inner product of (2-6) with $J_{1} J_{2} Y$. Suppose $v=3$. Taking the inner product of (2-6) with $J_{i} Y$ and $i \neq k$, we obtain

$$
\left\langle a_{i}, J_{k} Y\right\rangle\|Y\|^{2}=\left\langle a_{j}, Y\right\rangle\left\langle J_{i} J_{k} J_{j}, Y\right\rangle,
$$

where $\{i, j, k\}=\{1,2,3\}$. It follows that the polynomial $\left\langle J_{i} J_{k} J_{j} Y, Y\right\rangle$ is divisible by $\|Y\|^{2}$. Since the operator $J_{i} J_{k} J_{j}$ is symmetric and orthogonal, it equals $\tilde{\varepsilon}$ id, with $\tilde{\varepsilon}= \pm 1$; hence $J_{1} J_{2}=\varepsilon J_{3}$ with $\varepsilon= \pm 1$. Then $-J_{k} a_{i}=\tilde{\varepsilon} a_{j}$, so $J_{i} a_{j}=$ $-\tilde{\varepsilon} J_{i} J_{k} a_{i}=-\tilde{\varepsilon} J_{i} J_{k} a_{i}=J_{j} a_{i}$. Therefore for all $i, j$ such that $\{i, j, k\}=\{1,2,3\}$, 
we have $a_{j}=J_{i} v$ and $a_{i}=J_{j} v$, and we can assume that $v \neq 0$, since otherwise $a_{i}=a_{j}=0$.

Now suppose $v>3$ and let $L=\operatorname{Span}\left(a_{j}\right)$. It follows from (2-6) that if $Y \perp L$, then $J_{k} Y \perp L$, so $L$ is $J_{k}$-invariant. Polarizing (2-6) we obtain

$$
\sum_{j \neq k}\left(\left\langle a_{j}, J_{k} X\right\rangle J_{j} Y+\left\langle a_{j}, X\right\rangle J_{k} J_{j} Y\right)+\sum_{j \neq k}\left(\left\langle a_{j}, J_{k} Y\right\rangle J_{j} X+\left\langle a_{j}, Y\right\rangle J_{k} J_{j} X\right)=0 .
$$

It follows that, for all $X \perp L$ and all $Y \in \mathbb{R}^{n}$,

$$
\sum_{j \neq k}\left(\left\langle a_{j}, J_{k} Y\right\rangle J_{j} X+\left\langle a_{j}, Y\right\rangle J_{k} J_{j} X\right)=0
$$

that is, with $u(Y)=\sum_{j \neq k}\left\langle a_{j}, J_{k} Y\right\rangle e_{j}$ and $v(Y)=\sum_{j \neq k}\left\langle a_{j}, Y\right\rangle e_{j}$, we have that $J_{u(Y)} X=-J_{k} J_{v(Y)} X$. Note that $u(Y)$ and $v(Y)$ are perpendicular to $e_{k}$. Now, fix an arbitrary $Y \in \mathbb{R}^{n}$ and choose a unit vector $w$ perpendicular to $u(Y), v(Y)$ and $e_{k}$ in $\mathbb{R}^{v}$ (this is possible since $v>3$ ). Then $J_{w} J_{u(Y)} X=-J_{w} J_{k} J_{v(Y)} X$, so $\left\langle J_{w} J_{k} J_{v(Y)} X, X\right\rangle=0$ for all $X \in L^{\perp}$. If $v(Y) \neq 0$, the operator $\|v(Y)\|^{-1} J_{w} J_{k} J_{v(Y)}$ is symmetric and orthogonal, so the maximal dimension of its isotropic subspace is $n / 2<n-(v-1)=\operatorname{dim} L^{\perp}$ (the inequality follows from Lemma 2.4(ii)), which is a contradiction. Hence $v(Y)=0$ for all $Y \in \mathbb{R}^{n}$, so all the $a_{j}$ are zeros.

(3) We first prove the lemma assuming $2 v \leq n$. In this case, the proof closely follows the arguments in the proof of [N 2003, Lemma 3.1].

Let $Y_{0} \in T_{x} N^{n}$ be a unit vector. Since $2 v \leq n$, there exists a unit vector $E \in$ $T_{x} N^{n}$ that is not in the range of the map $\Phi: S^{\nu-1} \times S^{\nu-1} \rightarrow S^{n-1}, \Phi(u, v) \mapsto$ $J_{u} J_{v} Y_{0}$. Then $\mathscr{g} E \cap \mathscr{g} Y_{0}=0$. It follows that on some neighborhood $U^{\prime}$ of $x$, there exist smooth unit vector fields $Y$ and $E_{n}$ such that $E_{n}(x)=E, \quad Y(x)=Y_{0}$ and $\mathscr{g} E_{n} \cap \mathscr{F} Y=0$ at every point $y \in \mathcal{U}^{\prime}$. By assumption, the $v$-dimensional distribution $\mathscr{g} E_{n}$ is smooth, so we can choose $v$ smooth orthonormal sections $E_{1}, \ldots, E_{v}$ of it, and then define anticommuting almost Hermitian structures $\tilde{J}_{\alpha}$ on $\mathcal{U}^{\prime}$ satisfying $\tilde{J}_{\alpha} E_{n}=E_{\alpha}$ by setting $\tilde{J}_{\alpha}=\sum_{\beta=1}^{v} a_{\alpha \beta} J_{\beta}$, where $\left(a_{\alpha \beta}\right)$ is the $v \times v$ orthogonal matrix given by $a_{\alpha \beta}=\left\langle E_{\alpha}, J_{\beta} E_{n}\right\rangle$.

Let $E_{v+1}, \ldots, E_{n-1}$ be orthonormal vector fields on $U^{\prime}$ such that $E_{1}, \ldots, E_{n}$ is an orthonormal frame, and for a vector field $X$ on $\mathcal{U}^{\prime}$, let $\tilde{J} X$ denote the $n \times v$ matrix whose column vectors are $\tilde{J}_{1} X, \ldots, \tilde{J}_{v} X$ relative to the frame $E_{1}, \ldots, E_{n}$. Then $(\tilde{J} X)^{t} \tilde{J} X=\|X\|^{2} I_{v}$ and all the $v \times v$ minors of the matrix $\tilde{J} X$ are smooth functions on $\mathcal{U}^{\prime}$. Moreover, the entries of the matrices $\tilde{J} E_{i}$ for $i=1, \ldots, n$ are the rearranged entries of the matrices $\tilde{J}_{\alpha}$ for $\alpha=1, \ldots, v$ relative to the basis $\left\{E_{i}\right\}$, so to prove that the $\tilde{J}_{\alpha}$ are smooth it suffices to show that all the entries of the matrices $\tilde{J} E_{i}$ are smooth (on a possibly smaller neighborhood). Write $\tilde{J} E_{i}=\left(\begin{array}{c}K_{i} \\ P_{i}\end{array}\right)$, where $K_{i}$ and $P_{i}$ are respectively $v \times v$ and $(n-v) \times v$ matrix-valued functions on $U^{\prime}$; 
note that $\tilde{J} E_{n}=\left(\begin{array}{c}I_{v} \\ 0\end{array}\right)$. For an arbitrary $t \in \mathbb{R}$, all the $v \times v$ minors of the matrix

$$
\tilde{J}\left(E_{i}+t E_{n}\right)=\left(\begin{array}{c}
K_{i}+t I_{v} \\
P_{i}
\end{array}\right)
$$

are smooth. For every entry $\left(P_{i}\right)_{k \alpha}$, where $k=v+1, \ldots, n$ and $\alpha=1, \ldots, v$, the coefficient of $t^{\nu-1}$ in the $v \times v$ minor of $\tilde{J}\left(E_{i}+t E_{n}\right)$ consisting of $v-1$ out of the first $v$ rows (omitting the $\alpha$-th row) and the $k$-th row is $\pm\left(P_{i}\right)_{k \alpha}$, so all the entries of all the $P_{i}$ are smooth.

For the vector field $Y$ defined above, write $\tilde{J} Y=\left(\begin{array}{l}K \\ P\end{array}\right)$. Since $P=\sum_{i=1}^{n}\left\langle Y, E_{i}\right\rangle P_{i}$, all the entries of $P$ are smooth on $U^{\prime}$. Moreover, since $\mathscr{I} \cap \mathscr{I} E_{n}=0$, the spans of the vector columns of the matrices $\tilde{J} Y$ and $\tilde{J} E_{n}=\left(\begin{array}{c}I_{\nu} \\ 0\end{array}\right)$ have trivial intersection, so rk $P=v$ at every point $y \in U^{\prime}$. Therefore we can choose the rows $v+1 \leq$ $b_{1}<\cdots<b_{v} \leq n$ of the matrix $P$ at the point $x$ so that the corresponding minor $P_{(b)}=P_{b_{1} \ldots b_{v}}$ is nonzero. Then the same minor $P_{(b)}$ is nonzero on a (possibly smaller) neighborhood $U \subset U^{\prime}$ of $x$. Taking all the $v \times v$ minors of $\tilde{J} Y$ consisting of $v-1$ out of $v$ rows of $P_{(b)}$ and one row of $K$, we obtain that all the entries of $K$ are smooth on $U$. Moreover, for an arbitrary $t \in \mathbb{R}$, all the $v \times v$ minors of the matrix

$$
\tilde{J}\left(t E_{i}+Y\right)=\left(\begin{array}{c}
t K_{i}+K \\
t P_{i}+P
\end{array}\right)
$$

are smooth. Computing the coefficient of $t$ in all the $v \times v$ minors of $\tilde{J}\left(t E_{i}+Y\right)$ consisting of $v-1$ out of $v$ rows of $\left(t P_{i}+P\right)_{(b)}$ and one row of $t K_{i}+K$, and using the fact that all the entries of $K, P$ and $P_{i}$ are smooth on $U$, we obtain that all the entries of $K_{i}$ are also smooth on $U$. Therefore all the entries of all the matrices $\tilde{J} E_{i}$ are smooth on $U$; hence the anticommuting almost Hermitian structures $\tilde{J}_{\alpha}$ are also smooth on $U$.

Since $v$ and $n$ must satisfy inequality (2-3) (and hence those of Lemma 2.4), the above proof works in all the cases except when $n=4$ and $v=3$ and when $n=8$ and $v=5,6,7$. The former case is easy: Taking any smooth orthonormal frame $E_{i}$ on a neighborhood of $x$ and defining $\tilde{J}_{\alpha}=\sum_{\beta=1}^{3} a_{\alpha \beta} J_{\beta}$ with the orthogonal $3 \times 3$ matrix $\left(a_{\alpha \beta}\right)$ given by $a_{\alpha \beta}=\left\langle E_{\alpha}, J_{\beta} E_{4}\right\rangle$, we see that all the entries of the $\tilde{J}_{\alpha}$ relative to the basis $E_{i}$ are \pm 1 and 0 .

The proof in the cases that $n=8$ and $v=5,6,7$ is based on the fact that, except when $v=3$ and $J_{1} J_{2}= \pm J_{3}$, any set of anticommuting almost Hermitian structures $J_{1}, \ldots, J_{v}$ on $\mathbb{R}^{8}$ can be complemented by almost Hermitian structures $J_{v+1}, \ldots, J_{7}$ to a set $J_{1}, \ldots, J_{7}$ of anticommuting almost Hermitian structures on $\mathbb{R}^{8}$ (this is Lemma 2.6(1)).

If $n=8$ and $v=7$, choose an arbitrary smooth almost Hermitian structure $J_{7}$ on some neighborhood $U$ of $x$ and complement it by anticommuting almost Hermitian 
structures $J_{1}, \ldots, J_{6}$ at every point of $U$. Then for every smooth nowhere vanishing vector field $X$ on $U, \operatorname{Span}\left(J_{1} X, \ldots, J_{6} X\right)=\left(\operatorname{Span}\left(X, J_{7} X\right)\right)^{\perp}$ is a smooth distribution. This reduces the case $n=8$ and $v=7$ to the case $n=8$ and $v=6$.

Let $n=8$ and $v=6$, and let $J_{7}$ be an almost Hermitian structure complementing $J_{1}, \ldots, J_{6}$ at every point $x \in N^{n}$. Using the first part of the proof (or the fact that $J_{7} X$ spans the one-dimensional smooth distribution $\left(\operatorname{Span}\left(J_{1} X, \ldots, J_{6} X\right) \oplus \mathbb{R} X\right)^{\perp}$ for every nonvanishing smooth vector field $X$ ) we can assume that $J_{7}$ is smooth on a neighborhood $U$ of $x \in N^{n}$. Choose a smooth orthonormal frame $E_{1}, \ldots, E_{8}$ on (a possibly smaller neighborhood) $U$ such that the matrix of $J_{7}$ relative to $E_{i}$ is $\left(\begin{array}{cc}0 & I_{4} \\ -I_{4} & 0\end{array}\right)$ and define the almost Hermitian structure $\tilde{J}_{6}$ on $\mathcal{U}$ by

$$
\tilde{J}_{6} E_{2}=E_{1}, \quad \tilde{J}_{6} E_{4}=E_{3}, \quad \tilde{J}_{6} E_{6}=-E_{5}, \quad \tilde{J}_{6} E_{8}=-E_{7} .
$$

Then $J_{7}$ and $\tilde{J}_{6}$ anticommute; hence we can complement them by almost Hermitian structures $J_{1}^{\prime}, \ldots, J_{5}^{\prime}$ on $U$ so that $J_{1}^{\prime}, \ldots, J_{5}^{\prime}, \tilde{J}_{6}, J_{7}$ are anticommuting almost Hermitian structures. Moreover, since both $J_{7}$ and $\tilde{J}_{6}$ are smooth on $U$, the fivedimensional distribution $\operatorname{Span}\left(J_{1}^{\prime} X, \ldots, J_{5}^{\prime} X\right)=\left(\operatorname{Span}\left(X, J_{7} X, \tilde{J}_{6} X\right)\right)^{\perp}$ is smooth for every smooth nowhere vanishing vector field $X$ on $U$. This reduces the case $n=8$ and $v=6$ to the case $n=8$ and $v=5$. Indeed, if $\tilde{J}_{1}, \ldots, \tilde{J}_{5}$ are smooth anticommuting almost Hermitian structures on $U$ such that $\operatorname{Span}\left(\tilde{J}_{1} X, \ldots, \tilde{J}_{5} X\right)=$ $\operatorname{Span}\left(J_{1}^{\prime} X, \ldots, J_{5}^{\prime} X\right)$ for every vector field $X$, then $\tilde{J}_{1}, \ldots, \tilde{J}_{5}, \tilde{J}_{6}$ are the required almost Hermitian structures, since

$$
\begin{aligned}
\operatorname{Span}\left(\tilde{J}_{1} X, \ldots, \tilde{J}_{6} X\right) & =\operatorname{Span}\left(J_{1}^{\prime} X, \ldots, J_{5}^{\prime} X, \tilde{J}_{6} X\right) \\
& =\left(\operatorname{Span}\left(X, J_{7} X\right)\right)^{\perp}=\operatorname{Span}\left(J_{1} X, \ldots, J_{6} X\right),
\end{aligned}
$$

for every vector field $X$ on $U$, and $\tilde{J}_{6}$ anticommutes with every $\tilde{J}_{\alpha}$ for $\alpha=1, \ldots, 5$, since it anticommutes with every $J_{\alpha}^{\prime}$ for $\alpha=1, \ldots, 5$.

Let $n=8$ and $v=5$. Let $J_{6}$ and $J_{7}$ be anticommuting almost Hermitian structures complementing $J_{1}, \ldots, J_{5}$ at every point $x \in N^{n}$. Since $\operatorname{Span}\left(J_{6} X, J_{7} X\right)=$ $\left(\operatorname{Span}\left(J_{1} X, \ldots, J_{5} X\right)\right)^{\perp}$, we can choose such $J_{6}$ and $J_{7}$ to be smooth on a neighborhood $U$ of $x \in N^{n}$, by the first part of the proof. Choose a smooth orthonormal frame $E_{1}, \ldots, E_{8}$ on (a possibly smaller neighborhood) $U$ as follows. First choose an arbitrary smooth unit vector field $E_{1}$ on $U$. The vector fields $J_{6} E_{1}$ and $J_{7} E_{1}$ are orthonormal; set $E_{2}=-J_{6} E_{1}, E_{3}=-J_{7} E_{1}$. The unit vector field $J_{6} J_{7} E_{1}$ is orthogonal to $E_{1}, J_{6} E_{1}$ and $J_{7} E_{1}$; set $E_{4}=-J_{6} J_{7} E_{1}$. Choose an arbitrary smooth unit section $E_{5}$ of the smooth distribution $\left(\operatorname{Span}\left(E_{1}, E_{2}, E_{3}, E_{4}\right)\right)^{\perp}$ on $\mathcal{U}$. That distribution is both $J_{6^{-}}$and $J_{7}$-invariant, so we can set, similar to above, $E_{6}=J_{6} E_{5}, \quad E_{7}=J_{7} E_{5}$ and $E_{8}=-J_{6} J_{7} E_{5}$. Now define the almost Hermitian structure $\tilde{J}_{5}$ on $U$ whose matrix in the frame $E_{i}$ is $\left(\begin{array}{cc}0 & I_{4} \\ -I_{4} & 0\end{array}\right)$. Then $\tilde{J}_{5}, J_{6}$ and $J_{7}$

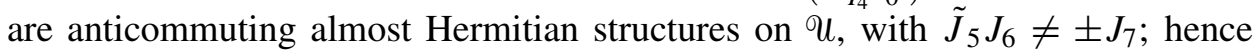


we can complement them by almost Hermitian structures $J_{1}^{\prime}, \ldots, J_{4}^{\prime}$ on $U$ in such a way that $J_{1}^{\prime}, \ldots, J_{4}^{\prime}, \tilde{J}_{5}, J_{6}, J_{7}$ are anticommuting almost Hermitian structures. Moreover, since $\tilde{J}_{5}, J_{6}$ and $J_{7}$ are smooth on $U$, the four-dimensional distribution $\operatorname{Span}\left(J_{1}^{\prime} X, \ldots, J_{4}^{\prime} X\right)=\left(\operatorname{Span}\left(X, \tilde{J}_{5} X, J_{6} X, J_{7} X\right)\right)^{\perp}$ is smooth for every smooth nowhere vanishing vector field $X$ on $\mathcal{U}$. By the first part of the proof, we can find smooth anticommuting almost Hermitian structures $\tilde{J}_{1}, \ldots, \tilde{J}_{4}$ on (a possibly smaller) neighborhood $U$ such that $\operatorname{Span}\left(\tilde{J}_{1} X, \ldots, \tilde{J}_{4} X\right)=\operatorname{Span}\left(J_{1}^{\prime} X, \ldots, J_{4}^{\prime} X\right)$ for every vector field $X$. Then $\tilde{J}_{1}, \ldots, \tilde{J}_{4}, \tilde{J}_{5}$ are the required almost Hermitian structures, since

$$
\begin{aligned}
\operatorname{Span}\left(\tilde{J}_{1} X, \ldots, \tilde{J}_{5} X\right) & =\operatorname{Span}\left(J_{1}^{\prime} X, \ldots, J_{4}^{\prime} X, \tilde{J}_{5} X\right) \\
& =\left(\operatorname{Span}\left(X, J_{6} X, J_{7} X\right)\right)^{\perp}=\operatorname{Span}\left(J_{1} X, \ldots, J_{5} X\right)
\end{aligned}
$$

for every vector field $X$ on $U$, and $\tilde{J}_{5}$ anticommutes with every $\tilde{J}_{\alpha}$ for $\alpha=1,2,3,4$, since it anticommutes with every $J_{\alpha}^{\prime}$ for $\alpha=1,2,3,4$.

\section{Conformally Osserman manifolds: Proof of Theorem 1.3}

Let $M^{n}$ be a smooth conformally Osserman Riemannian manifold with $n \neq 3,4$. If $n=2$, the manifold is locally conformally flat, so we can assume that $n>4$. Combining [N 2005, Proposition 1 and the penultimate paragraph of the proof of Theorems 1 and 2] with [N 2004, Proposition 1] and [N 2006, Proposition 2.1], we obtain that the Weyl tensor of $M^{n}$ has a Clifford structure for all $n \neq 16$, and also for $n=16$ provided the Jacobi operator $W_{X}$ has an eigenvalue of multiplicity at least 9 (note that the Jacobi operator of any Osserman algebraic curvature tensor on $\mathbb{R}^{16}$ has an eigenvalue of multiplicity at least 7 , for topological reasons). In the latter case, $W$ has a Clifford structure $\operatorname{Cliff}(v)$, with $v \leq 6$, at every point on $M^{n}$.

To prove Theorem 1.3 it therefore suffices to prove the following theorem.

Theorem 3.1. Let $M^{n}$ be a connected smooth Riemannian manifold whose Weyl tensor at every point $x \in M^{n}$ has a Clifford structure $\operatorname{Cliff}(v(x))$. Suppose that $n>4$, and additionally that $v(x) \leq 4$ if $n=16$. Then there exists a space $M_{0}^{n}$ from the list $\mathbb{R}^{n}, \mathbb{C} P^{n / 2}, \mathbb{C} H^{n / 2}, \mathbb{H} P^{n / 4}, \mathbb{H} H^{n / 4}$ (Euclidean space and the rank-one symmetric spaces with their standard metrics) such that $M^{n}$ is locally conformally equivalent to $M_{0}^{n}$.

Note that by Theorem 3.1, every point of $M^{n}$ has a neighborhood conformally equivalent to a domain of the same "model space". Also note that the theorem says something also in the case $n=16$, whereas Theorem 3.1 does not.

We start with a sketch of the proof of Theorem 3.1. First, we show that the Clifford structure for the Weyl tensor can be chosen locally smooth on an open, dense subset $M^{\prime} \subset M^{n}$ (see Lemma 3.2 for the precise statement). To simplify 
the form of the curvature tensor $R$ of $M^{n}$, we combine the $\lambda_{0}$-part of $W$ (from (2-1)) with the difference $R-W$, so that $R$ has the form (3-1) for some smooth symmetric operator field $\rho$ at every point of $M^{\prime}$. The technical core of the proof is Lemmas 3.5 and 3.6, which establish various identities for the covariant derivatives of $\rho$, the $J_{i}$ and the $\eta_{i}$, using the second Bianchi identity for the curvature tensor of the form (3-1). Lemma 3.6 treats the case $(n, v)=(8,7)$ and uses the octonion arithmetic; Lemma 3.5 treats all the other cases, and uses the fact that $v$ is small compared to $n$ - see Lemma 2.4. It follows from the identities of Lemma 3.5 and Lemma 3.6 that, unless the Weyl tensor vanishes, the metric on $M^{\prime}$ can be locally changed to a conformal one whose curvature tensor again has the form (3-1), but with the two additional features: First, all the $\eta_{i}$ are locally constant, and second, $\rho$ is a Codazzi tensor, that is, $\left(\nabla_{X} \rho\right) Y=\left(\nabla_{Y} \rho\right) X$. By the result of [Derdziński and Shen 1983], exterior products of the eigenspaces of a symmetric Codazzi tensor are invariant under the curvature operator on the two-forms. Using that, we prove in Lemma 3.7 that $\rho$ must be a multiple of the identity, so, by (3-1), $M^{\prime}$ is locally conformally equivalent to an Osserman manifold. The affirmative answer to the Osserman conjecture in the cases for $n$ and $v$ considered in Theorem 3.1, given by [N 2003, Theorem 1.2], implies that $M^{\prime}$ is locally conformally equivalent to one of the spaces listed in Theorem 3.1. This proves Theorem 3.1 at the generic points. To prove Theorem 3.1 globally, we first show, using Lemma 3.9, that $M$ splits into a disjoint union of a closed subset $M_{0}$, on which the Weyl tensor vanishes, and nonempty open connected subsets $M_{\alpha}$, each of which is locally conformal to one of the rank-one symmetric spaces $\mathbb{C} P^{n / 2}, \mathbb{C} H^{n / 2}, \mathbb{H} P^{n / 4}, \mathbb{H} H^{n / 4}$. On every $M_{\alpha}$, the conformal factor $f$ is a well-defined positive smooth function. Assuming that there exists at least one $M_{\alpha}$ and that $M_{0} \neq \varnothing$, we show in Lemma 3.10 that there exists a point $x_{0} \in M_{0}$ on the boundary of a geodesic ball $B \subset M_{\alpha}$ such that both $f(x)$ and $\nabla f(x)$ tend to zero when $x \rightarrow x_{0}$ for $x \in B$. Then the positive function $u=f^{(n-2) / 4}$ satisfies the elliptic equation (3-31) in $B$, with $\lim _{x \rightarrow x_{0}, x \in B} u(x)=0$; hence by the boundary point theorem, the limiting value of the inner derivative of $u$ at $x_{0}$ must be positive. This contradiction implies that either $M=M_{0}$ or $M=M_{\alpha}$. Proof of Theorem 3.1. For $n>4$, let $M^{n}$ be a connected smooth Riemannian manifold whose Weyl tensor at every point has a Clifford structure. Define the function $N: M^{n} \rightarrow \mathbb{N}$ so that $N(x)$ is the number of distinct eigenvalues of the Jacobi operator $W_{X}$ associated to the Weyl tensor, where $X$ is an arbitrary nonzero vector from $T_{x} M^{n}$. Since the Weyl tensor is Osserman, $N(x)$ is well defined. Moreover, since the set of symmetric operators having no more than $N_{0}$ distinct eigenvalues is closed in the linear space of symmetric operators on $\mathbb{R}^{n}$, the function $N(x)$ is lower semicontinuous, that is, every subset $\left\{x: N(x) \leq N_{0}\right\}$ is closed in $M^{n}$. Let $M^{\prime}$ be the set of points where the function $N(x)$ is continuous. It is easy to see that $M^{\prime}$ is an open and dense (but possibly disconnected) subset of $M^{n}$. The 
following lemma shows that the Clifford structure for the Weyl tensor is locally smooth on every connected component of $M^{\prime}$.

Lemma 3.2. For $n>4$, let $M^{n}$ be a smooth Riemannian manifold whose Weyl tensor has a Clifford structure at every point. If $n=16$, we additionally require that at every point $x \in M^{16}$, the Weyl tensor has a Clifford structure $\operatorname{Cliff}(v(x))$ with $v(x) \neq 8$.

Let $M^{\prime}$ be the (open, dense) subset of $M^{n}$, at the points of which the number of distinct eigenvalues of the Jacobi operator associated to the Weyl tensor of $M^{n}$ is locally constant. Then for every $x \in M^{\prime}$, there exists a neighborhood $U=U(x)$, a number $v \geq 0$, smooth functions $\eta_{1}, \ldots, \eta_{v}: \mathcal{U} \rightarrow \mathbb{R} \backslash\{0\}$, a smooth symmetric linear operator field $\rho$, and smooth anticommuting almost Hermitian structures $J_{i}$ for $i=1, \ldots, v$, on $U$ such that the curvature tensor of $M^{n}$ has the form

$$
\begin{aligned}
& R(X, Y) Z=\langle X, Z\rangle \rho Y+\langle\rho X, Z\rangle Y-\langle Y, Z\rangle \rho X-\langle\rho Y, Z\rangle X \\
& \quad+\sum_{i=1}^{v} \eta_{i}\left(2\left\langle J_{i} X, Y\right\rangle J_{i} Z+\left\langle J_{i} Z, Y\right\rangle J_{i} X-\left\langle J_{i} Z, X\right\rangle J_{i} Y\right),
\end{aligned}
$$

for all $y \in U$ and $X, Y, Z \in T_{y} M^{n}$. Moreover, if $n=8$, then the curvature tensor has the form (3-1) either with $v=3$ and $J_{1} J_{2}= \pm J_{3}$, or with $v=7$ for all $y \in \mathcal{U}$.

Proof. Let $X$ be a smooth unit vector field on $M^{n}$. Since the Weyl tensor $W$ is a smooth Osserman algebraic curvature tensor, the characteristic polynomial of $W_{X \mid X^{\perp}}$ (of the restriction of the Jacobi operator $W_{X}$ to the subspace $X^{\perp}$ ) does not depend on $X$ and is a well-defined smooth map $p: M^{n} \rightarrow \mathbb{R}_{n-1}[t], y \mapsto p_{y}(t)$, where $\mathbb{R}_{n-1}[t]$ is the $(n-1)$-dimensional affine space of polynomials of degree $n-1$ with leading term $(-t)^{n-1}$. Since all the roots of $p_{y}(t)$ are real and the number of different roots is constant on every connected component of $M^{\prime}$, the eigenvalues $\mu_{0}, \mu_{1}, \ldots, \mu_{l}$ of $W_{X \mid X^{\perp}}$ are smooth functions and their multiplicities $m_{0}, m_{1}, \ldots, m_{l}$ are constant on every connected component of $M^{\prime}$ (we chose the labeling so that $m_{0}=\max \left\{m_{0}, m_{1}, \ldots, m_{l}\right\}$.

First consider the case $n \neq 8$. The Weyl tensor has a Clifford structure given by (2-1) at every point of $M^{\prime}$. By Lemma 2.4, for $n>4$ with $n \neq 8,16$, we have $n-1-v>v$ for any Clifford structure on $\mathbb{R}^{n}$. By (2-3), we have $v \leq 8$ for $n=16$, so by assumption, the inequality $n-1-v>v$ also holds for $n=$ 16. Then the biggest multiplicity of an eigenvalue of $W_{X \mid X^{\perp}}$ is $n-1-v$; see Remark 2.3. So $v=n-1-m_{0}$ is constant and the function $\lambda_{0}=\mu_{0}$ is smooth on every connected component of $M^{\prime}$. Moreover, for every smooth unit vector field $X$ on $M^{\prime}$ and every $i=1, \ldots, l$, the $\mu_{i}$-eigendistribution of $W_{X \mid X^{\perp}}$ is $\operatorname{Span}_{j: \lambda_{0}+3 \eta_{j}=\mu_{i}}\left(J_{j} X\right)$. Since $\lambda_{0}$ and $\mu_{i}$ are smooth functions on every connected component of $M^{\prime}$, so is $\eta_{j}$. Moreover, on every connected component of $M^{\prime}$, every distribution $\operatorname{Span}_{j: \lambda_{0}+3 \eta_{j}=\mu_{i}}\left(J_{j} X\right)$ is smooth and has a constant dimension $m_{i}$ for 
any nowhere vanishing smooth vector field $X$. By Lemma 2.7(3), there exists a neighborhood $U_{i}(x)$ and smooth anticommuting almost Hermitian structures $\tilde{J}_{j}$ (for $j$ such that $\lambda_{0}+3 \eta_{j}=\mu_{i}$ ) on $U_{i}(x)$ such that

$$
\operatorname{Span}_{j: \lambda_{0}+3 \eta_{j}=\mu_{i}}\left(J_{j} X\right)=\operatorname{Span}_{j: \lambda_{0}+3 \eta_{j}=\mu_{i}}\left(\tilde{J}_{j} X\right) .
$$

Let $\widetilde{W}$ be the algebraic curvature tensor on $u=\bigcap_{i=1}^{l} U_{i}(x)$ with the Clifford structure $\operatorname{Cliff}\left(v ; \tilde{J}_{1}, \ldots, \tilde{J}_{v} ; \lambda_{0}, \eta_{1}, \ldots, \eta_{v}\right)$. Then $v=n-1-m_{0}$ is constant and all the $\tilde{J}_{i}, \eta_{i}$ and $\lambda_{0}$ are smooth on $\mathcal{U}$. Moreover, for every unit vector field $X$ on $U$, the Jacobi operators $\widetilde{W}_{X}$ and $W_{X}$ have the same eigenvalues and the same eigenspaces by construction; hence $\widetilde{W}_{X}=W_{X}$, which implies $\widetilde{W}=W$.

Now consider the case $n=8$. By Lemma 2.6, at every point $x \in M^{\prime}$, the Weyl tensor either has a Cliff(3) structure with $J_{1} J_{2}=J_{3}$ or a Cliff(7) structure (but not both). Since on every connected component $M_{\alpha}$ of $M^{\prime}$ the eigenvalues of the operator $W_{X \mid X^{\perp}}$ with $X \neq 0$ have constant number and multiplicity, Remark 2.3 implies that the only case when $M_{\alpha}$ may potentially contain points of both kinds is when one of the eigenvalues of $W_{X \mid X^{\perp}}$ with $X \neq 0$ on $M_{\alpha}$ has multiplicity 4 and the Clifford structure at every point $x \in M_{\alpha}$ is either

$$
\operatorname{Cliff}\left(3 ; J_{1}, J_{2}, J_{3} ; \lambda_{0}, \eta_{1}, \eta_{2}, \eta_{3}\right)
$$

with $J_{1} J_{2}=J_{3}$, or

$$
\operatorname{Cliff}\left(7 ; J_{1}, \ldots, J_{7} ; \lambda_{0}-3 \xi, \eta_{1}+\xi, \eta_{2}+\xi, \eta_{3}+\xi, \xi, \xi, \xi, \xi\right),
$$

where $\eta_{1}, \eta_{2}, \eta_{3} \neq 0$ (some of them can be equal) and $\xi \neq-\eta_{i}, 0$. The eigenvalues of $W_{X \mid X^{\perp}}$ with $\|X\|=1$ at every point $x \in M_{\alpha}$ are $\lambda_{0}$, of multiplicity 4 , and $\lambda_{0}+3 \eta_{i}$. Let $X$ be an arbitrary nowhere vanishing smooth vector field on a neighborhood $U \subset M_{\alpha}$ of a point $x \in M_{\alpha}$. Then the four-dimensional eigendistribution of $W_{X \mid X^{\perp}}$ corresponding to the eigenvalue of multiplicity 4 is smooth; hence its orthogonal complement, the distribution $\operatorname{Span}\left(J_{1} X, J_{2} X, J_{3} X\right)$, is also smooth. By Lemma 2.7(3), there are smooth anticommuting almost Hermitian structures $\tilde{J}_{1}, \tilde{J}_{2}, \tilde{J}_{3}$ such that $\operatorname{Span}\left(\tilde{J}_{1} X, \tilde{J}_{2} X, \tilde{J}_{3} X\right)=\operatorname{Span}\left(J_{1} X, J_{2} X, J_{3} X\right)$ on (a possibly smaller) neighborhood $U$. By Lemma $2.7(1)$ with $F(X)=\tilde{J}_{i} X$, every $\tilde{J}_{i}$ is a linear combination of the $J_{j}: \tilde{J}_{i}=\sum_{j=1}^{3} a_{i j} J_{j}$, and moreover, the matrix $\left(a_{i j}\right)$ must be orthogonal, since the $\tilde{J}_{i}$ are anticommuting almost Hermitian structures. It follows that $\tilde{J}_{1} \tilde{J}_{2} \tilde{J}_{3}= \pm J_{1} J_{2} J_{3}$. The operator on the left side is smooth on $U$, the one on the right side is $\pm \mathrm{id}_{\mathbb{R}^{8}}$ at the points where the Clifford structure is Cliff(3) with $J_{1} J_{2}=J_{3}$, and is symmetric with trace zero at the points where the Clifford structure is Cliff(7), which follows from the identity $J_{4}\left(J_{1} J_{2} J_{3}\right) J_{4}=J_{1} J_{2} J_{3}$. Therefore all the points of $U$ either have a $\operatorname{Cliff(3)}$ structure with $J_{1} J_{2}=J_{3}$ or a $\operatorname{Cliff}(7)$ structure. In both cases, the Clifford structure for $W$ can be taken to be smooth: 
In the first, this follows from the arguments similar to those in the first part of the proof, since $v<n-1-v$; in the second, we apply Lemma 2.7(3) to every eigendistribution of $W_{X \mid X^{\perp}}$.

Thus for any $x \in M^{\prime}$, the Weyl tensor on a neighborhood $U=U(x)$ has the form (2-1), with a constant $v$ and smooth $\lambda_{0}, \eta_{i}$ and $J_{i}$. Then the curvature tensor has the form (3-1) with the operator $\rho$ given by

$$
\rho=\frac{1}{n-2} \operatorname{Ric}+\left(\frac{\lambda_{0}}{2}-\frac{\text { scal }}{2(n-1)(n-2)}\right) \mathrm{id},
$$

where Ric is the Ricci operator and scal is the scalar curvature. Since $\lambda_{0}$ is a smooth function, the operator field $\rho$ is also smooth.

Remark 3.3. In fact, the proof shows that if an algebraic curvature tensor field $\mathscr{R}$ has a Clifford structure at every point of a Riemannian manifold (and $v \neq 8$ when $n=16$ ), then it has a Clifford structure of the same class of differentiability as $\mathscr{R}$ on a neighborhood of every generic point of the manifold.

Remark 3.4. It follows from Lemma 2.6(1) (in fact, from Equation (2-4)) that, in the case $n=8$ and $v=7$ we can replace $\rho$ by $\rho-\frac{3}{2} f$ id and $\eta_{i}$ by $\eta_{i}+f$ in (3-1) without changing $R$, where $f$ is an arbitrary smooth function on $U$. If we want the resulting Clifford structure to be Cliff(7), we additionally require that $\eta_{i}+f$ is nowhere zero.

Let $x \in M^{\prime}$, and let $U=U(x)$ be its neighborhood defined in Lemma 3.2. By the second Bianchi identity, $\left(\nabla_{U} R\right)(X, Y) Y+\left(\nabla_{Y} R\right)(U, X) Y+\left(\nabla_{X} R\right)(Y, U) Y=0$. Substituting $R$ from (3-1) and using the fact that the operators $J_{i}$ and their covariant derivatives are skew-symmetric and the operator $\rho$ and its covariant derivatives are symmetric we get

$$
\begin{gathered}
+\langle U, Y\rangle\left(\left(\nabla_{Y} \rho\right) X-\left(\nabla_{X} \rho\right) Y\right)+\left\langle\left(\nabla_{Y} \rho\right) U-\left(\nabla_{U} \rho\right) Y, Y\right\rangle X \\
+\left\langle\left(\nabla_{X} \rho\right) Y-\left(\nabla_{Y} \rho\right) X, Y\right\rangle U+\left\langle\left(\nabla_{U} \rho\right) X-\left(\nabla_{X} \rho\right) U, Y\right\rangle Y \\
+\sum_{i=1}^{v} 3\left(X\left(\eta_{i}\right)\left\langle J_{i} Y, U\right\rangle-U\left(\eta_{i}\right)\left\langle J_{i} Y, X\right\rangle\right) J_{i} Y \\
+\sum_{i=1}^{v} Y\left(\eta_{i}\right)\left(2\left\langle J_{i} U, X\right\rangle J_{i} Y+\left\langle J_{i} Y, X\right\rangle J_{i} U-\left\langle J_{i} Y, U\right\rangle J_{i} X\right) \\
+\sum_{i=1}^{v} \eta_{i}\left(\left(3\left\langle\left(\nabla_{U} J_{i}\right) X, Y\right\rangle+3\left\langle\left(\nabla_{X} J_{i}\right) Y, U\right\rangle+2\left\langle\left(\nabla_{Y} J_{i}\right) U, X\right\rangle\right) J_{i} Y\right. \\
+3\left\langle J_{i} X, Y\right\rangle\left(\nabla_{U} J_{i}\right) Y+3\left\langle J_{i} Y, U\right\rangle\left(\nabla_{X} J_{i}\right) Y+2\left\langle J_{i} U, X\right\rangle\left(\nabla_{Y} J_{i}\right) Y \\
+\left\langle\left(\nabla_{Y} J_{i}\right) Y, X\right\rangle J_{i} U+\left\langle J_{i} Y, X\right\rangle\left(\nabla_{Y} J_{i}\right) U \\
\left.-\left\langle\left(\nabla_{Y} J_{i}\right) Y, U\right\rangle J_{i} X-\left\langle J_{i} Y, U\right\rangle\left(\nabla_{Y} J_{i}\right) X\right)=0 .
\end{gathered}
$$$$
\langle X, Y\rangle\left(\left(\nabla_{U} \rho\right) Y-\left(\nabla_{Y} \rho\right) U\right)+\|Y\|^{2}\left(\left(\nabla_{X} \rho\right) U-\left(\nabla_{U} \rho\right) X\right)
$$ 
Taking the inner product of (3-2) with $X$ and assuming $X, Y$ and $U$ to be orthogonal, we obtain

$$
\|X\|^{2}\langle Q(Y), U\rangle+\|Y\|^{2}\langle Q(X), U\rangle
$$

$$
\begin{aligned}
& +\sum_{i=1}^{v} 3\left(X\left(\eta_{i}\right)\left\langle J_{i} Y, U\right\rangle-Y\left(\eta_{i}\right)\left\langle J_{i} X, U\right\rangle-U\left(\eta_{i}\right)\left\langle J_{i} Y, X\right\rangle\right)\left\langle J_{i} Y, X\right\rangle \\
& +\sum_{i=1}^{v} 3 \eta_{i}\left(\left(2\left\langle\left(\nabla_{U} J_{i}\right) X, Y\right\rangle+\left\langle\left(\nabla_{X} J_{i}\right) Y, U\right\rangle+\left\langle\left(\nabla_{Y} J_{i}\right) U, X\right\rangle\right)\left\langle J_{i} Y, X\right\rangle\right. \\
& \left.-\left\langle J_{i} Y, U\right\rangle\left\langle\left(\nabla_{X} J_{i}\right) X, Y\right\rangle-\left\langle J_{i} X, U\right\rangle\left\langle\left(\nabla_{Y} J_{i}\right) Y, X\right\rangle\right)=0,
\end{aligned}
$$

where $Q: \mathbb{R}^{n} \rightarrow \mathbb{R}^{n}$ is the quadratic map defined by

$$
\langle Q(X), U\rangle=\left\langle\left(\nabla_{X} \rho\right) U-\left(\nabla_{U} \rho\right) X, X\right\rangle .
$$

Note that $\langle Q(X), X\rangle=0$.

Lemma 3.5. Under the assumptions of Lemma 3.2, let $x \in M^{\prime}$ and let $\mathcal{U}$ be the corresponding neighborhood of $x$. Suppose that if $n=8$, then $v=3$ and $J_{1} J_{2}=J_{3}$ on $U$, and if $n=16$, then $v \leq 4$. For every point $y \in U$, identify $T_{y} M^{n}$ with Euclidean $\mathbb{R}^{n}$ via a linear isometry.

(i) There exist $m_{i}, b_{i j} \in \mathbb{R}^{n}$ with $i, j=1, \ldots, v$ such that for all $X, Y, U \in \mathbb{R}^{n}$ and all $i, j=1, \ldots, v$,

$$
\begin{aligned}
& Q(Y)=3 \sum_{k=1}^{v}\left\langle m_{k}, Y\right\rangle J_{k} Y, \\
&\left(\nabla_{X} J_{i}\right) X=\eta_{i}^{-1}\left(\|X\|^{2} m_{i}-\left\langle m_{i}, X\right\rangle X\right)+\sum_{j=1}^{v}\left\langle b_{i j}, X\right\rangle J_{j} X, \\
& b_{i j}+b_{j i}=\eta_{i}^{-1} J_{j} m_{i}+\eta_{j}^{-1} J_{i} m_{j}, \\
& \nabla \eta_{i}=2 J_{i} m_{i}, \\
& \sum_{j \neq i}\left(\left\langle\eta_{i} b_{i j}+\eta_{j} b_{j i}, J_{i} Y\right\rangle J_{j} Y+\left\langle\eta_{i} b_{i j}+\eta_{j} b_{j i}, Y\right\rangle J_{i} J_{j} Y\right)=0 .
\end{aligned}
$$

(ii) These equations hold:

$$
\begin{gathered}
\left(\nabla_{Y} \rho\right) U-\left(\nabla_{U} \rho\right) Y=\sum_{i=1}^{v}\left(2\left\langle J_{i} Y, U\right\rangle m_{i}-\left\langle m_{i}, Y\right\rangle J_{i} U+\left\langle m_{i}, U\right\rangle J_{i} Y\right) \\
b_{i j}\left(3-\eta_{i} \eta_{j}^{-1}\right)+b_{j i}\left(3-\eta_{j} \eta_{i}^{-1}\right)=0 \quad \text { for } i \neq j \\
J_{i} m_{i}=\eta_{i} p \quad \text { for } i=1, \ldots, v \text { and some } p \in \mathbb{R}^{n}
\end{gathered}
$$

Proof. (i) We split the proof of these assertions into two cases: the exceptional case, when either $n=6$ and $v=1$, or $n=12, v=3$ and $J_{1} J_{2}= \pm J_{3}$, or $n=8$, $v=3$ and $J_{1} J_{2}=J_{3}$, and the generic case, consisting of all the other Clifford structures considered in the lemma. 
Generic case. From (3-3) we obtain

$$
\|X\|^{-2}\langle Q(X), U\rangle+\|Y\|^{-2}\langle Q(Y), U\rangle=0
$$

for all $X \perp \mathscr{I} Y, X, Y \perp \mathscr{I}$, and $X, Y, U \neq 0$.

We want to show that $\langle Q(X), U\rangle=0$ for all $X \perp \mathscr{I} U$. This is immediate when $n>3 v+3$. Indeed, $\operatorname{codim}(\mathscr{G} U+\mathscr{I} X)>v+1$ for any $U \neq 0$ and any unit $X \perp \mathscr{I} U$, so we can choose unit vectors $Y_{1}, Y_{2} \perp \mathscr{I} U+\mathscr{I} X$ such that $Y_{1} \perp \mathscr{I} Y_{2}$. Then (3-7) implies that $\langle Q(X), U\rangle=-\left\langle Q\left(Y_{1}\right), U\right\rangle=\left\langle Q\left(Y_{2}\right), U\right\rangle=-\langle Q(X), U\rangle$.

Consider the case $n \leq 3 v+3$. By Lemma 2.4(i), this could only happen when $n=12$ and $v=3$ or $n=24$ and $v=7$ (for the pairs $(n, v)$ belonging to the generic case), and in both cases, $n=3 v+3$. Choose and fix an arbitrary $U \neq 0$ and consider the quadratic form $q(X)=\langle Q(X), U\rangle$ defined on the $(2 v+2)$-dimensional space $L=(\mathscr{I} U)^{\perp}$. Suppose $q \neq 0$. By (3-7), the restriction of $q$ to the unit sphere of $L$ is not a constant, so it attains its maximum (respectively minimum) on a great sphere $S_{1}$ (respectively $S_{2}$ ). The subspaces $L_{1}$ and $L_{2}$ defined by $S_{1}$ and $S_{2}$ are orthogonal. Moreover by (3-7), we have $L_{2} \supset(\Im X)^{\perp} \cap L$ for any nonzero $X \in L_{1}$, which implies that $\operatorname{dim} L_{2} \geq v+1$. Similarly $\operatorname{dim} L_{1} \geq v+1$, so $\operatorname{dim} L_{1}=\operatorname{dim} L_{2}=v+1$ since $L_{1} \perp L_{2}$, and $L=L_{1} \oplus L_{2}$. It follows that $q(X)=c\left(\left\|\pi_{1} X\right\|^{2}-\left\|\pi_{2} X\right\|^{2}\right)$ for some $c>0$, where $\pi_{i}: L \rightarrow L_{i}$ is the orthogonal projection. Also, $L_{2}=(\Phi X)^{\perp} \cap L$ for all nonzero $X \in L_{1}$, which means that the subspace $L_{1}=L_{2}^{\perp} \cap L$ (and similarly

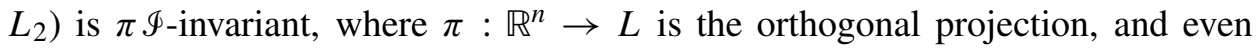
furthermore $\pi \Im X=L_{\alpha}$ for every nonzero $X \in L_{\alpha}$ for $\alpha=1$, 2, by dimension count. Let $X=X_{1}+X_{2}$ and $Y=Y_{1}+Y_{2} \in L$, where $X_{\alpha}=\pi_{\alpha} X$ and $Y_{\alpha}=\pi_{\alpha} Y$. The condition $Y \perp \mathscr{I} X$ is equivalent to

$$
\left\langle X_{1}, Y_{1}\right\rangle+\left\langle X_{2}, Y_{2}\right\rangle=\left\langle\pi J_{i} X_{1}, Y_{1}\right\rangle+\left\langle\pi J_{i} X_{2}, Y_{2}\right\rangle=0 \quad \text { for all } i=1, \ldots v .
$$

Take arbitrary orthonormal bases for $L_{1}$ and for $L_{2}$ and let $M_{\alpha}\left(X_{\alpha}\right)$ for $\alpha=1,2$ be the $(v+1) \times(v+1)$ matrix whose columns relative to the chosen basis for $L_{\alpha}$ are $X_{\alpha}, \pi J_{1} X_{\alpha}, \ldots, \pi J_{v} X_{\alpha}$. Then $Y \perp \mathscr{I} X$ if and only if $M_{1}\left(X_{1}\right)^{t} Y_{1}=-M_{2}\left(X_{2}\right)^{t} Y_{2}$. Since for $\alpha=1,2$, and any nonzero $X_{\alpha} \in L_{\alpha}$, the columns of $M_{\alpha}\left(X_{\alpha}\right)$ span $L_{\alpha}$, we obtain $Y_{2}=-\left(M_{2}\left(X_{2}\right)^{t}\right)^{-1} M_{1}\left(X_{1}\right)^{t} Y_{1}$ for any $X_{2} \neq 0$. Then, since

$$
q(X)=c\left(\left\|X_{1}\right\|^{2}-\left\|X_{2}\right\|^{2}\right) \quad \text { and } \quad q(Y)=c\left(\left\|Y_{1}\right\|^{2}-\left\|Y_{2}\right\|^{2}\right),
$$

Equation (3-7) implies $\left\|Y_{1}\right\|^{2}\left\|X_{1}\right\|^{2}-\left\|Y_{2}\right\|^{2}\left\|X_{2}\right\|^{2}=0$, so

$$
\left\|Y_{1}\right\|^{2}\left\|X_{1}\right\|^{2}-\left\|\left(M_{2}\left(X_{2}\right)^{t}\right)^{-1} M_{1}\left(X_{1}\right)^{t} Y_{1}\right\|^{2}\left\|X_{2}\right\|^{2}=0
$$

for any $X_{1}, Y_{1} \in L_{1}$ and any nonzero $X_{2} \in L_{2}$. It follows that

$$
\left\|X_{1}\right\|^{2}\left(M_{1}\left(X_{1}\right)^{t} M_{1}\left(X_{1}\right)\right)^{-1}=\left\|X_{2}\right\|^{2}\left(M_{2}\left(X_{2}\right)^{t} M_{2}\left(X_{2}\right)\right)^{-1}
$$


for any nonzero $X_{\alpha} \in L_{\alpha}$. Thus for some positive definite symmetric $(v+1) \times(v+1)$ matrix $T$, we have

$$
M_{\alpha}\left(X_{\alpha}\right)^{t} M_{\alpha}\left(X_{\alpha}\right)=\left\|X_{\alpha}\right\|^{2} T
$$

for all $X_{\alpha} \in L_{\alpha}$ with $\alpha=1,2$. Then for any $X=X_{1}+X_{2} \in L$ with $X_{\alpha} \in L_{\alpha}$, and any $i=1, \ldots, v$,

$$
\begin{aligned}
\left\|\pi J_{i} X\right\|^{2}=\left\|\pi J_{i} X_{1}\right\|^{2}+\left\|\pi J_{i} X_{2}\right\|^{2} & =\left(M_{1}\left(X_{1}\right)^{t} M_{1}\left(X_{1}\right)+M_{2}\left(X_{2}\right)^{t} M_{2}\left(X_{2}\right)\right)_{i i} \\
& =T_{i i}\left(\left\|X_{1}\right\|^{2}+\left\|X_{2}\right\|^{2}\right)=T_{i i}\|X\|^{2} .
\end{aligned}
$$

On the other hand, $\pi J_{i} X=J_{i} X-\|U\|^{-2} \sum_{j=1}^{v}\left\langle J_{i} X, J_{j} U\right\rangle J_{j} U$ for any $X \in L$, so $\left\|\pi J_{i} X\right\|^{2}=\|X\|^{2}-\|U\|^{-2} \sum_{j=1}^{v}\left\langle J_{i} X, J_{j} U\right\rangle^{2}$. It follows that

$$
\|X\|^{2}\|U\|^{2}\left(1-T_{i i}\right)=\sum_{j=1}^{\nu}\left\langle J_{i} X, J_{j} U\right\rangle^{2}=\sum_{j=1}^{\nu}\left\langle X, J_{i} J_{j} U\right\rangle^{2}
$$

for an arbitrary $X \in L$. Since $\operatorname{dim} L=2 v+2>v$, we can choose a nonzero $X \in L$ orthogonal to the $v$ vectors $J_{i} J_{j} U$, for $j=1, \ldots, v$. This implies $T_{i i}=1$, and so $X \perp J_{i} J_{j} U$, for all $i, j=1, \ldots, v$ and all $X \in L=(\mathscr{I} U)^{\perp}$. Therefore $J_{i} J_{j} U \in \mathscr{I} U$ for all $i, j=1, \ldots, v$ and all $U \in \mathbb{R}^{n}$ for which the quadratic form $q(X)=\langle Q(X), U\rangle$ defined on $(\mathscr{G U})^{\perp}$ is nonzero. If this is true for at least one $U$, then this is true for a dense subset of $\mathbb{R}^{n}$, which implies that $J_{i} J_{j} U \in \mathscr{I} U$ for all $i, j=1, \ldots, v$ and all $U \in \mathbb{R}^{n}$. Then by Lemma 2.7(1), $J_{i} J_{j} U=\sum_{k=1}^{v} a_{i j k} J_{k} U$ for $i \neq j$ for some constants $a_{i j k}$, which implies that $\left\langle J_{k} J_{i} J_{j} U, U\right\rangle=a_{i j k}\|U\|^{2}$, so for all triples of pairwise distinct $i, j, k$, the symmetric operator $J_{k} J_{i} J_{j}$ on $\mathbb{R}^{n}$ is a multiple of the identity. This is impossible when $v>3$ (since for $l \neq i, j, k$, the operator $J_{l} J_{k} J_{i} J_{j}$ must be orthogonal and symmetric). The only remaining cases are $n=12$ and $v=3$, with $J_{1} J_{2} J_{3}= \pm \mathrm{id}$, and $n=6$ and $v=1$, which are considered under the exceptional case below.

Therefore $\langle Q(X), U\rangle=0$ for $X \perp \mathscr{I} U$, so $Q(X) \in \mathscr{I} X$ for all $X \in \mathbb{R}^{n}$. By Lemma 2.7(1) (and the fact that $\langle Q(X), X\rangle=0$ ), this implies (3-5a) for some vectors $m_{i} \in \mathbb{R}^{n}$.

To prove (3-5b) and (3-5c), we first show that for an arbitrary $X \neq 0$, there is a dense subset of the $Y$ in $(\mathscr{I} X)^{\perp}$ such that $\mathscr{\mathscr { S }} X \cap \mathscr{g} Y=0$. This follows from the dimension count (compare to [N 2003, Lemma 3.2(1)]). For $X \neq 0$, define the cone $\mathscr{C} X=\left\{J_{u} J_{v} X: u, v \in \mathbb{R}^{v}\right\}$; see (2-2). Since

$$
\operatorname{dim} \mathscr{C} X \leq 2 v-1<n-(v+1)=\operatorname{dim}(\Im X)^{\perp},
$$

where the inequality in the middle follows from Lemma 2.4(i), the complement to $\mathscr{C} X$ is dense in $(\mathscr{I} X)^{\perp}$. This complement is the required subset, since the condition $Y \notin \mathscr{C} X$ is equivalent to $\mathscr{\mathscr { S }} X \cap \mathscr{g} Y=0$. Substituting such $X, Y$ into (3-3) we obtain 
by $(3-5 a)$

$$
\begin{aligned}
\sum_{i=1}^{v}\left(\|X\|^{2}\left\langle m_{i}, Y\right\rangle-\eta_{i}\left\langle\left(\nabla_{X} J_{i}\right) X, Y\right\rangle\right) J_{i} Y & \\
& +\sum_{i=1}^{v}\left(\|Y\|^{2}\left\langle m_{i}, X\right\rangle-\eta_{i}\left\langle\left(\nabla_{Y} J_{i}\right) Y, X\right\rangle\right) J_{i} X=0 .
\end{aligned}
$$

Since $\mathscr{\mathscr { X }} \cap \mathscr{\mathscr { S }} Y=0$, all the coefficients vanish, so

$$
\|X\|^{2}\left\langle m_{i}, Y\right\rangle-\eta_{i}\left\langle\left(\nabla_{X} J_{i}\right) X, Y\right\rangle=0
$$

for all $X \in \mathbb{R}^{n}$, all $i=1, \ldots, \nu$, and all $Y$ from a dense subset of $(\mathscr{g} X)^{\perp}$, which implies that $\left(\nabla_{X} J_{i}\right) X-\eta_{i}^{-1}\|X\|^{2} m_{i} \in \mathscr{I} X$ for all $X \in \mathbb{R}^{n}$. Equation (3-5b) then follows from Lemma 2.7(1). Equation (3-5c) follows from (3-5b) and the fact that $\left\langle\left(\nabla_{X} J_{i}\right) X, J_{j} X\right\rangle+\left\langle\left(\nabla_{X} J_{j}\right) X, J_{i} X\right\rangle=0$.

To prove (3-5d) and (3-5e), substitute $X=J_{k} Y$ and $U \perp X, Y$ into (3-3). Since $\left\langle J_{i} Y, X\right\rangle=\|Y\|^{2} \delta_{i k}$, the first term in the second sum equals

$$
3 \eta_{k}\left(2\left\langle\left(\nabla_{U} J_{k}\right) X, Y\right\rangle+\left\langle\left(\nabla_{X} J_{k}\right) Y, U\right\rangle+\left\langle\left(\nabla_{Y} J_{k}\right) U, X\right\rangle\right)\|Y\|^{2} .
$$

Since $J_{k}$ is orthogonal and skew-symmetric,

$$
\left\langle\left(\nabla_{U} J_{k}\right) X, Y\right\rangle=\left\langle\left(\nabla_{U} J_{k}\right) J_{k} Y, Y\right\rangle=-\left\langle J_{k}\left(\nabla_{U} J_{k}\right) Y, Y\right\rangle=\left\langle\left(\nabla_{U} J_{k}\right) Y, J_{k} Y\right\rangle=0 .
$$

Next,

$$
\begin{aligned}
\left\langle\left(\nabla_{Y} J_{k}\right) U, X\right\rangle & =-\left\langle\left(\nabla_{Y} J_{k}\right) J_{k} Y, U\right\rangle=\left\langle J_{k}\left(\nabla_{Y} J_{k}\right) Y, U\right\rangle \\
& =\left\langle\left(\eta_{k}^{-1}\|Y\|^{2} J_{k} m_{k}+\sum_{j=1}^{v}\left\langle b_{k j}, Y\right\rangle J_{k} J_{j} Y, U\right\rangle\right.
\end{aligned}
$$

by (3-5b). Similarly, since $Y=-J_{k} X$, it follows from (3-5b) that

$$
\begin{aligned}
\left\langle\left(\nabla_{X} J_{k}\right) Y, U\right\rangle & =\left\langle J_{k}\left(\nabla_{X} J_{k}\right) X, U\right\rangle \\
& =\left\langle J_{k}\left(\eta_{k}^{-1}\left(\|X\|^{2} m_{k}-\left\langle m_{k}, X\right\rangle X\right)+\sum_{j=1}^{v}\left\langle b_{k j}, X\right\rangle J_{j} X\right), U\right\rangle \\
& =\left\langle\eta_{k}^{-1}\|Y\|^{2} J_{k} m_{k}+\sum_{j \neq k}\left\langle b_{k j}, J_{k} Y\right\rangle J_{j} Y-\left\langle b_{k k}, J_{k} Y\right\rangle J_{k} Y, U\right\rangle .
\end{aligned}
$$

Substituting this into (3-3) and using (3-5a) and (3-5b), we obtain after simplification

$$
\begin{aligned}
\|Y\|^{2}\left(\left\langle 2 J_{k} m_{k}, U\right\rangle\right. & \left.-U\left(\eta_{k}\right)\right) \\
& +\sum_{j=1}^{v}\left\langle\eta_{k} b_{k j}+\eta_{j} b_{j k},\left\langle J_{j} Y, U\right\rangle J_{k} Y+\left\langle J_{k} J_{j} Y, U\right\rangle Y\right\rangle=0 .
\end{aligned}
$$

By [N 2003, Lemma 3.2(3)] for all $U \in \mathbb{R}^{n}$, we can find a nonzero $Y$ such that $U \perp \mathscr{J} Y+\mathscr{J} J_{k} Y$. Substituting such a $Y$ into (3-8) proves (3-5d). Then (3-8) simplifies to (3-5e). 
Exceptional case. Here either $n=6$ and $v=1$, or $n=12, v=3$ and $J_{1} J_{2}= \pm J_{3}$, or $n=8$ and $v=3$ and $J_{1} J_{2}=J_{3}$.

In all these cases, the Clifford structure has the " $J^{2}$ property" that $\mathscr{I} \mathscr{I} X=$ $\mathscr{I} \Phi=\Phi X$ for every $X \in \mathbb{R}^{n}$. In particular, if $Y \perp \mathscr{I} X$, then $\mathscr{Y} \perp \mathscr{I} X$.

Substitute $X=J_{k} U$ and $Y \perp \mathscr{I} X=\mathscr{I} U$ into (3-2) and take the inner product of the resulting equation with $J_{k} Y$. Using the $J^{2}$ property and the fact that $\left\langle\left(\nabla_{Y} J_{k}\right) U, J_{k} U\right\rangle=\left\langle\left(\nabla_{Y} J_{k}\right) Y, J_{k} Y\right\rangle=0$, we get

$-J_{k}\left(\left(\nabla_{J_{k}} \rho\right) U-\left(\nabla_{U} \rho\right) J_{k} U\right)+2\|U\|^{2} \nabla \eta_{k}+3 \eta_{k}\left(\left(\nabla_{U} J_{k}\right) J_{k} U-\left(\nabla_{J_{k} U} J_{k}\right) U\right) \in \mathscr{I} U$.

The expression $F(U)$ on the left side is a quadratic map from $\mathbb{R}^{n}$ to itself. By Lemma 2.7(1), $F(U)$ is a linear combination of $U, J_{1} U, \ldots, J_{v} U$ whose coefficients are linear forms of $U$. In particular, the cubic polynomial $\left\langle F(U), J_{k} U\right\rangle$ must be divisible by $\|U\|^{2}$. Since $J_{k}$ is orthogonal and skew-symmetric,

$$
\left\langle\left(\nabla_{U} J_{k}\right) J_{k} U-\left(\nabla_{J_{k} U} J_{k}\right) U, J_{k} U\right\rangle=0,
$$

so there exists a vector $m_{k} \in \mathbb{R}^{n}$ such that

$$
\left\langle\left(\nabla_{J_{k} U} \rho\right) U-\left(\nabla_{U} \rho\right) J_{k} U, U\right\rangle=-3\|U\|^{2}\left\langle m_{k}, U\right\rangle .
$$

It follows that the quadratic map $Q$ defined by (3-4) satisfies

$$
\left\langle Q(U), J_{k} U\right\rangle=3\|U\|^{2}\left\langle m_{k}, U\right\rangle \text { for all } U \in \mathbb{R}^{n} \text { and all } k=1, \ldots, v .
$$

Since $\langle Q(U), U\rangle=0$, we can define a quadratic map $T: \mathbb{R}^{n} \rightarrow \mathbb{R}^{n}$ such that for all $U \in \mathbb{R}^{n}$,

$$
Q(U)=T(U)+3 \sum_{k=1}^{v}\left\langle m_{k}, U\right\rangle J_{k} U \quad \text { and } \quad T(U) \perp \mathscr{I} U .
$$

Taking $U=J_{k} X, X, U \perp \mathscr{I} Y$ in (3-3) and using (3-9) we obtain

$$
-J_{k} T(Y)+3\|Y\|^{2} m_{k}-3 \eta_{k}\left(\nabla_{Y} J_{k}\right) Y \in \mathscr{I} Y .
$$

From Lemma 2.7(1) it follows that the expression on the left side is a linear combination of $Y, J_{1} Y, \ldots, J_{v} Y$ whose coefficients are linear forms of $Y$, so for some vectors $b_{i j} \in \mathbb{R}^{n}$,

$$
\left(\nabla_{Y} J_{i}\right) Y=\eta_{i}^{-1}\left(m_{i}\|Y\|^{2}-\left\langle m_{i}, Y\right\rangle Y\right)-\left(3 \eta_{i}\right)^{-1} J_{i} T(Y)+\sum_{j=1}^{\nu}\left\langle b_{i j}, Y\right\rangle J_{j} Y .
$$

Since $\left\langle\left(\nabla_{Y} J_{i}\right) Y, J_{j} Y\right\rangle$ is antisymmetric in $i$ and $j$ and $J_{i} T(Y) \perp \Phi Y$ by (3-9) and the $J^{2}$ property, the $b_{i j}$ satisfy (3-5c).

Take $X=J_{k} Y$ and $U \perp \mathscr{I} Y=\mathscr{I} X$ in (3-3). Since $\left\langle\left(\nabla_{U} J_{k}\right) J_{k} Y, Y\right\rangle=0$,

$$
\left\langle\left(\nabla_{Y} J_{k}\right) U, X\right\rangle=-\left\langle\left(\nabla_{Y} J_{k}\right) J_{k} Y, U\right\rangle=\left\langle J_{k}\left(\nabla_{Y} J_{k}\right) Y, U\right\rangle,
$$


and similarly $\left\langle\left(\nabla_{X} J_{k}\right) Y, U\right\rangle=-\left\langle\left(\nabla_{X} J_{k}\right) J_{k} X, U\right\rangle=\left\langle J_{k}\left(\nabla_{X} J_{k}\right) X, U\right\rangle$, we obtain from (3-9) and (3-10) after simplification that

$$
2 T(Y)+2 T\left(J_{k} Y\right)-3\|Y\|^{2}\left(\nabla \eta_{k}-2 J_{k} m_{k}\right) \in \Phi Y .
$$

In case $n=6$ and $v=1$, we can prove the remaining identities (3-5a), (3-5b), (3-5d) and (3-5e) of Lemma 3.5(i) as follows. Taking in (3-3) nonzero $X, Y, U$ such that the subspaces $\Phi X, \Phi Y$ and $\mathscr{I} U$ are mutually orthogonal we obtain by (3-9)

$$
\|X\|^{-2}\langle T(X), U\rangle+\|Y\|^{-2}\langle T(Y), U\rangle=0,
$$

which is, essentially, (3-7). Replacing $Y$ by $J_{1} Y$ and using (3-11) we get

$$
2 T(X)+3\|X\|^{2}\left(\nabla \eta_{1}-2 J_{1} m_{1}\right) \in \mathscr{I} X .
$$

The same is true with $X$ replaced by $J_{1} X$. Then by (3-11), $\nabla \eta_{1}-2 J_{1} m_{1} \in \mathscr{I} X$ for all $X \in \mathbb{R}^{6}$, so $\nabla \eta_{1}-2 J_{1} m_{1}=0$, which is (3-5d). Then $T(X) \in \Phi X$; hence $T(X)=0$, since $T(X) \perp \mathscr{X}$ by (3-9). Now (3-5a) follows from (3-9), (3-5b) follows from (3-10), and (3-5e) is trivially satisfied, as $v=1$.

In the cases $n=8,12, v=3$ and $J_{1} J_{2}=J_{3}$ (if $J_{1} J_{2}=-J_{3}$, we replace $J_{3}$ by $-J_{3}$ without changing the curvature tensor (3-1)), we argue as follows. Adding (3-11) with $k=1$ and with $k=2$ and then subtracting (3-11) with $k=3$ and $Y$ replaced by $J_{1} Y$ we get

$$
4 T(Y)-3\|Y\|^{2}\left(\left(\nabla \eta_{1}-2 J_{1} m_{1}\right)+\left(\nabla \eta_{2}-2 J_{2} m_{2}\right)-\left(\nabla \eta_{3}-2 J_{3} m_{3}\right)\right) \in \mathscr{I} Y .
$$

This remains true under a cyclic permutation of the indices 1,2,3, which implies $\left(\nabla \eta_{k}-2 J_{k} m_{k}\right)-\left(\nabla \eta_{i}-2 J_{i} m_{i}\right) \in \mathscr{I} Y$ for all $i, k=1,2,3$ and all $Y \in \mathbb{R}^{n}$. Then $\nabla \eta_{k}-2 J_{k} m_{k}=\nabla \eta_{i}-2 J_{i} m_{i}=4 V / 3$ for some vector $V \in \mathbb{R}^{n}$, and $T(Y)-\|Y\|^{2} V$

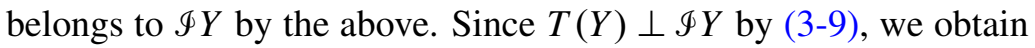

$$
T(Y)=\|Y\|^{2} V-\langle Y, V\rangle Y-\sum_{i=1}^{3}\left\langle J_{i} Y, V\right\rangle J_{i} Y,
$$

so

$$
\begin{aligned}
\nabla \eta_{i} & =2 J_{i} m_{i}+\frac{4}{3} V, \\
Q(Y) & =\|Y\|^{2} V-\langle Y, V\rangle Y+\sum_{j=1}^{3}\left\langle 3 m_{j}+J_{j} V, Y\right\rangle J_{j} Y, \\
\left(\nabla_{Y} J_{i}\right) Y & =\left(3 \eta_{i}\right)^{-1}\left(\|Y\|^{2}\left(3 m_{i}-J_{i} V\right)-\left\langle 3 m_{i}-J_{i} V, Y\right\rangle Y\right. \\
& \left.+\sum_{j=1}^{3}\left\langle 3 \eta_{i} b_{i j}-J_{j} J_{i} V, Y\right\rangle J_{j} Y\right),
\end{aligned}
$$

where the second equation follows from (3-9) and the third from (3-10) and the fact that $J_{1} J_{2}=J_{3}$.

Substitute $X=J_{k} Y$ into (3-3) again, with an arbitrary $U \perp X, Y$. Using (3-12) and that the $J_{i}$ are skew-symmetric, orthogonal and anticommute, we obtain after 
simplification that

$$
\sum_{i=1}^{3}\left\langle 3 a_{i k}-2 J_{i} J_{k} V, J_{k} Y\right\rangle J_{i} Y+\sum_{i=1}^{3}\left\langle 3 a_{i k}-2 J_{i} J_{k} V, Y\right\rangle J_{k} J_{i} Y \in \operatorname{Span}\left(Y, J_{k} Y\right),
$$

where $a_{i k}=\eta_{k} b_{k i}+\eta_{i} b_{i k}$. Taking $k=1$ and using that $J_{1} J_{2}=J_{3}$, we get from the coefficient of $J_{2} Y$ that $3 J_{1} a_{12}-4 J_{2} V+3 a_{13}=0$, so $4 V=-3 J_{2} a_{13}+3 J_{3} a_{12}$. Cyclically permuting the indices $1,2,3$ and using that $a_{i k}=a_{k i}$, we get $V=0$, which implies (3-5e). Since $V=0$, equations (3-5a), (3-5d) and (3-5b) follow from (3-12).

(ii) By (3-4) and (3-5a),

$$
\left\langle\left(\nabla_{X} \rho\right) U-\left(\nabla_{U} \rho\right) X, X\right\rangle=3 \sum_{i=1}^{v}\left\langle m_{i}, X\right\rangle\left\langle J_{i} X, U\right\rangle \quad \text { for all } X, U \in \mathbb{R}^{n} .
$$

Polarizing this equation and using the fact that the covariant derivative of $\rho$ is symmetric, we obtain

$$
\begin{aligned}
& \left\langle\left(\nabla_{X} \rho\right) U, Y\right\rangle+\left\langle\left(\nabla_{Y} \rho\right) U, X\right\rangle-2\left\langle\left(\nabla_{U} \rho\right) Y, X\right\rangle \\
& \quad=3 \sum_{i=1}^{\nu}\left(\left\langle m_{i}, Y\right\rangle\left\langle J_{i} X, U\right\rangle+\left\langle m_{i}, X\right\rangle\left\langle J_{i} Y, U\right\rangle\right) .
\end{aligned}
$$

Subtracting the same equation with $Y$ and $U$ interchanged, we get

$$
\begin{aligned}
\left\langle\left(\nabla_{Y} \rho\right) U-\left(\nabla_{U} \rho\right) Y, X\right\rangle= & \sum_{i=1}^{v}\left(2\left\langle m_{i}, X\right\rangle\langle\right. \\
& \left.J_{i} Y, U\right\rangle \\
& \left.+\left\langle m_{i}, Y\right\rangle\left\langle J_{i} X, U\right\rangle-\left\langle m_{i}, U\right\rangle\left\langle J_{i} X, Y\right\rangle\right),
\end{aligned}
$$

which proves (3-6a).

To establish (3-6b), substitute $X \perp \mathscr{I} Y, U=J_{k} Y$ into (3-2). Using the equations of part (i) and (3-6a) we obtain after simplification that

$$
\begin{aligned}
3\left(\nabla_{X} J_{k}\right) Y- & \left(\nabla_{Y} J_{k}\right) X \\
& =-3 \eta_{k}^{-1}\left\langle m_{k}, Y\right\rangle X+\sum_{i=1}^{v} \eta_{k}^{-1}\left\langle\eta_{i} b_{i k}+2 \delta_{i k} J_{k} m_{k}, Y\right\rangle J_{i} X \quad \bmod (\Phi Y) .
\end{aligned}
$$

Subtracting thrice polarized Equation (3-5b) (with $i=k$ ) and solving for $\left(\nabla_{Y} J_{k}\right) X$, we get, for all $X \perp \mathscr{I} Y$,

$$
\left(\nabla_{Y} J_{k}\right) X=\sum_{i=1}^{\nu} \frac{1}{4} \eta_{k}^{-1}\left\langle 3 \eta_{k} b_{k i}-\eta_{i} b_{i k}-2 \delta_{i k} J_{k} m_{k}, Y\right\rangle J_{i} X \bmod (\Phi Y) .
$$

Choose $s \neq k$ and define the subset $S_{k s} \subset \mathbb{R}^{n} \oplus \mathbb{R}^{n}$ by

$$
S_{k s}=\left\{(X, Y): X, Y \neq 0 \text { and } X, J_{k} X, J_{s} X \perp \mathscr{Y} Y\right\} .
$$


It is easy to see that $(X, Y) \in S_{k s}$ if and only if $(Y, X) \in S_{k s}$ and that replacing $\mathscr{\&} Y$ by $\mathscr{I} Y$ in the definition of $S_{k s}$ gives the same set $S_{k s}$. Moreover, $\left\{X:(X, Y) \in S_{k s}\right\}$ (and hence $\left.\left\{Y:(X, Y) \in S_{k s}\right\}\right)$ spans $\mathbb{R}^{n}$. If $n=8, v=3$ and $J_{1} J_{2}=J_{3}$, this follows from the $J^{2}$-property; in all other cases, it follows from [N 2003, Lemma 3.2(4)]. For $(X, Y) \in S_{k s}$, take the inner product of (3-13) with $J_{s} X$. Since $\left\langle\left(\nabla_{Y} J_{k}\right) X, J_{s} X\right\rangle$ is antisymmetric in $k$ and $s$, we get $\left\langle\left(3-\eta_{k} \eta_{s}^{-1}\right) b_{k s}+\left(3-\eta_{s} \eta_{k}^{-1}\right) b_{s k}, Y\right\rangle=0$ for a set of $Y$ spanning $\mathbb{R}^{n}$. This proves (3-6b).

To prove (3-6c), we apply of Lemma 2.7(2) to (3-5e). If $v=1$, there is nothing to prove; in fact, if $v=1$ and $n \geq 8$, Theorem 3.1 follows from [Blažić and Gilkey 2004, Theorem 1.1]. If $\eta_{i} b_{i j}+\eta_{j} b_{j i}=0$ for all $i \neq j$, then by (3-6b), $b_{i j}+b_{j i}=0$ for all $i \neq j$, so $\eta_{i}^{-1} J_{j} m_{i}=-\eta_{j}^{-1} J_{i} m_{j}$ by (3-5c). Acting by $J_{i} J_{j}$ we obtain that the vector $\eta_{i}^{-1} J_{i} m_{i}$ is the same for all $i=1, \ldots, v$.

The only remaining possibility is $v=3, J_{1} J_{2}=J_{3}$ (if $J_{1} J_{2}=-J_{3}$ we can replace $J_{3}$ by $-J_{3}$ without changing the curvature tensor (3-1)), and $\eta_{k} b_{k i}+\eta_{i} b_{i k}=J_{j} v$ for all the triples $\{i, j, k\}=\{1,2,3\}$, where $v \neq 0$. We will show that this leads to a contradiction. Note that by (2-3), the existence of a Cliff(3) structure implies that $n$ is divisible by 4 , so $n \geq 8$ by the assumption of the lemma.

If $\eta_{i}=\eta_{k}$ for some $i \neq k$, then from (3-6b) and $\eta_{k} b_{k i}+\eta_{i} b_{i k}=J_{j} v$ it follows that $v=0$, a contradiction. Otherwise, if the $\eta_{i}$ are pairwise distinct, we get

$$
b_{i k}=\left(3 \eta_{i}-\eta_{k}\right)\left(4 \eta_{i}\left(\eta_{i}-\eta_{k}\right)\right)^{-1} J_{j} v \quad \text { for }\{i, j, k\}=\{1,2,3\} .
$$

Substituting this into (3-5c) and acting by $J_{j}$ on both sides, we get

$$
\eta_{i}^{-1} J_{i} m_{i}-\eta_{k}^{-1} J_{k} m_{k}=\frac{1}{4} \varepsilon_{i k}\left(\eta_{i}^{-1}+\eta_{k}^{-1}\right) v \quad \text { for }\{i, j, k\}=\{1,2,3\},
$$

where for $i \neq k$ we define $\varepsilon_{i k}= \pm 1$ by $J_{i} J_{k}=\varepsilon_{i k} J_{j}$. It is easy to see that $\varepsilon_{j k}=-\varepsilon_{j k}$ and $\varepsilon_{j k}=\varepsilon_{i j}$, where $\{i, j, k\}=\{1,2,3\}$. Then

$$
\sum_{i=1}^{3} \eta_{i}^{-1}=0 \quad \text { and } \quad \eta_{i}^{-1} J_{i} m_{i}=\frac{1}{12} \varepsilon_{j k}\left(\eta_{j}^{-1}-\eta_{k}^{-1}\right) v+w \quad \text { for some } w \in \mathbb{R}^{n} .
$$

It then follows from (3-5d) that $\nabla \eta_{i}=(1 / 6) \varepsilon_{j k} \eta_{i}\left(\eta_{j}^{-1}-\eta_{k}^{-1}\right) v+2 \eta_{i} w$, which implies

$$
\nabla \ln \left|\eta_{1} \eta_{2} \eta_{3}\right|=6 w \quad \text { and } \quad \nabla \ln \left|\eta_{i} \eta_{j}^{-1}\right|=-\frac{1}{2} \varepsilon_{i j} \eta_{k}^{-1} v .
$$

Let $u^{\prime} \subset U$ be a neighborhood of $x$ on which $\nabla \ln \left|\eta_{1} \eta_{2}^{-1}\right| \neq 0$. Then $v$ is a nowhere vanishing smooth vector field on $\mathcal{u}^{\prime}$. Multiplying the metric on $\mathcal{U}$ by a function $e^{f}$ changes neither the Weil tensor nor the $J_{i}$, and multiplies every $\eta_{i}$ by $e^{-f}$ and $\nabla$ acting on functions by $e^{-f}$. Taking $f=(1 / 3) \ln \left|\eta_{1} \eta_{2} \eta_{3}\right|$ we can assume that $w=0$ on $U^{\prime}$, so that $C=\eta_{1} \eta_{2} \eta_{3}$ is a constant. Then, since $\sum_{i=1}^{3} \eta_{i}^{-1}=0$, we get

$$
\nabla \eta_{i}= \pm \frac{1}{6} v \sqrt{1-4 C^{-1} \eta_{i}^{3}}
$$


It follows that $v=\nabla t$ for some smooth function $t: u^{\prime} \rightarrow \mathbb{R}$ such that $\eta_{i}=$ $-36 C \wp\left(t+c_{i}\right)$, where $\wp$ is the Weierstrass function satisfying

$$
\left(\frac{d}{d t} \wp(t)\right)^{2}=4 \wp(t)^{3}+6^{-6} C^{-2}
$$

and $c_{i} \in \mathbb{R}$. Summarizing these identities, we have pointwise pairwise nonequal functions $\eta_{i}: U^{\prime} \rightarrow \mathbb{R} \backslash\{0\}$ satisfying

$$
\begin{array}{rlrl}
v & =\nabla t \neq 0, & \nabla \eta_{i} & =\frac{1}{6} \varepsilon_{j k} \eta_{i}\left(\eta_{j}^{-1}-\eta_{k}^{-1}\right) v, \\
0 & =\sum_{i=1}^{3} \eta_{i}^{-1}, & C & =\mathrm{const}=\prod_{i=1}^{3} \eta_{i}, \\
m_{i} & =-\frac{1}{12} \varepsilon_{j k} \eta_{i}\left(\eta_{j}^{-1}-\eta_{k}^{-1}\right) J_{i} v, & b_{i i} & =\frac{1}{12} \varepsilon_{j k}\left(\eta_{j}^{-1}-\eta_{k}^{-1}\right) v, \\
b_{i j} & =\left(3 \eta_{i}-\eta_{j}\right)\left(4 \eta_{i}\left(\eta_{i}-\eta_{j}\right)\right)^{-1} J_{k} v,
\end{array}
$$

for $\{i, j, k\}=\{1,2,3\}$, where we used (3-5c) to compute $b_{i i}$. Then Equation (3-13) simplifies to

$$
\left(\nabla_{Y} J_{k}\right) X=\sum_{i \neq k} \frac{1}{2}\left(\eta_{k}-\eta_{i}\right)^{-1}\left\langle J_{j} v, Y\right\rangle J_{i} X \quad \bmod (\mathscr{Y} Y) \quad \text { for all } X \perp \mathscr{I} Y .
$$

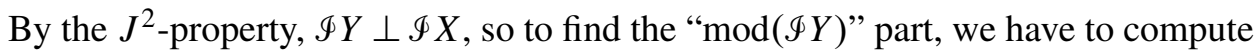
the inner products of $\left(\nabla_{Y} J_{k}\right) X$ with $Y, J_{1} Y, J_{2} Y$ and $J_{3} Y$. Since

$$
\begin{aligned}
\left\langle\left(\nabla_{Y} J_{k}\right) X, Y\right\rangle & =-\left\langle\left(\nabla_{Y} J_{k}\right) Y, X\right\rangle, \\
\left\langle\left(\nabla_{Y} J_{k}\right) X, J_{k} Y\right\rangle & =-\left\langle\left(\nabla_{Y} J_{k}\right) J_{k} Y, X\right\rangle=\left\langle J_{k}\left(\nabla_{Y} J_{k}\right) Y, X\right\rangle, \\
\left\langle\left(\nabla_{Y} J_{k}\right) X, J_{i} Y\right\rangle & =-\left\langle\left(\nabla_{Y} J_{k}\right) J_{i} Y, X\right\rangle=-\left\langle\left(\varepsilon_{k i}\left(\nabla_{Y} J_{j}\right)-J_{k}\left(\nabla_{Y} J_{i}\right)\right) Y, X\right\rangle
\end{aligned}
$$

(from $J_{k} J_{i}=\varepsilon_{k i} J_{j}$ ), these products can be found using (3-5b). Simplifying by (3-14) we get

$$
\begin{aligned}
\left(\nabla_{Y} J_{k}\right) X=\frac{1}{12} \varepsilon_{i j}\left(\eta_{i}^{-1}-\right. & \left.\eta_{j}^{-1}\right)\left(\left\langle J_{k} v, X\right\rangle Y+\langle v, X\rangle J_{k} Y\right) \\
& +\frac{1}{4} \eta_{k}^{-1} \sum_{i \neq k}\left\langle J_{j} v, X\right\rangle J_{i} Y+\sum_{i \neq k} \frac{1}{2}\left(\eta_{k}-\eta_{i}\right)^{-1}\left\langle J_{j} v, Y\right\rangle J_{i} X,
\end{aligned}
$$

for all $X \perp \mathscr{I} Y$, where $\{i, j, k\}=\{1,2,3\}$. To compute $\left(\nabla_{Y} J_{k}\right) X$ when $X \in \mathscr{I} Y$, we again use (3-5b) and the fact that, for $\{i, j, k\}=\{1,2,3\}$,

$$
\left(\nabla_{Y} J_{k}\right) J_{k}=-J_{k}\left(\nabla_{Y} J_{k}\right) \quad \text { and } \quad\left(\nabla_{Y} J_{k}\right) J_{i}=\varepsilon_{k i}\left(\nabla_{Y} J_{j}\right)-J_{k}\left(\nabla_{Y} J_{i}\right) .
$$

Simplifying by (3-14) and using the above equation we get after some calculation

$$
\begin{array}{r}
\left(\nabla_{Y} J_{k}\right) X=\frac{1}{12} \varepsilon_{i j}\left(\eta_{i}^{-1}-\eta_{j}^{-1}\right)\left(\left\langle J_{k} v, X\right\rangle Y+\langle v, X\rangle J_{k} Y-\langle X, Y\rangle J_{k} v-\left\langle X, J_{k} Y\right\rangle v\right) \\
+\frac{1}{4} \eta_{k}^{-1} \sum_{i \neq k}\left(\left\langle J_{j} v, X\right\rangle J_{i} Y-\left\langle J_{i} Y, X\right\rangle J_{j} v\right)+\sum_{i \neq k} \frac{1}{2}\left(\eta_{k}-\eta_{i}\right)^{-1}\left\langle J_{j} v, Y\right\rangle J_{i} X,
\end{array}
$$


for all $X, Y \in \mathbb{R}^{n}$, where $\{i, j, k\}=\{1,2,3\}$. For $a, b \in \mathbb{R}^{n}$, let $a \wedge b$ be the skewsymmetric operator defined by $(a \wedge b) X=\langle a, X\rangle b-\langle b, X\rangle a$. Then the above equation can be written in the form

$$
\begin{aligned}
\nabla_{Y} J_{k}=\frac{1}{12} \varepsilon_{i j}\left(\eta_{i}^{-1}-\eta_{j}^{-1}\right)\left(J_{k} v\right. & \left.\wedge Y+v \wedge J_{k} Y\right) \\
+ & \frac{1}{4} \eta_{k}^{-1} \sum_{i \neq k} J_{j} v \wedge J_{i} Y+\sum_{i \neq k} \frac{1}{2}\left(\eta_{k}-\eta_{i}\right)^{-1}\left\langle J_{j} v, Y\right\rangle J_{i},
\end{aligned}
$$

that is, for $\{i, j, k\}=\{1,2,3\}$,

$$
\begin{aligned}
\nabla_{Y} J_{k} & =\left[J_{k}, A Y\right], & A Y & =\sum_{i=1}^{3}\left(\frac{1}{2} \lambda_{i} J_{i} Y \wedge J_{i} v+\omega_{i}\left\langle J_{i} v, Y\right\rangle J_{i}\right), \\
\lambda_{i} & =\frac{1}{6} \varepsilon_{j k}\left(\eta_{j}^{-1}-\eta_{k}^{-1}\right), & \omega_{i} & =\frac{1}{4} \varepsilon_{j k}\left(\eta_{k}-\eta_{j}\right)^{-1}
\end{aligned}
$$

where we used that, for $\{i, j, k\}=\{1,2,3\}$,

$$
\left[J_{k}, a \wedge b\right]=J_{k} a \wedge b+a \wedge J_{k} b \quad \text { and } \quad\left[J_{k}, J_{i}\right]=2 \varepsilon_{k i} J_{j} .
$$

By the Ricci formula, $\nabla_{Z, Y}^{2} J_{k}-\nabla_{Y, Z}^{2} J_{k}=\left[J_{k}, R(Y, Z)\right]$, where the tensor field $\nabla^{2} J_{k}$ is defined by

$$
\nabla_{Z, Y}^{2} J_{k}=\nabla_{Z}\left(\nabla_{Y} J_{k}\right)-\nabla_{\nabla_{Z} Y} J_{k} \text { for vector fields } Y, Z \text { on } U^{\prime} .
$$

Since $\nabla_{Y} J_{k}=\left[J_{k}, A Y\right]$ by (3-15), this is equivalent to the fact that the operator $F(Y, Z)=\left(\nabla_{Z} A\right) Y-\left(\nabla_{Y} A\right) Z-[A Y, A Z]-R(Y, Z)$ commutes with all the $J_{s}$ for all $Y, Z \in \mathbb{R}^{n}$ and all $s=1,2,3$. By (3-1), we have

$$
R(Y, Z)=Y \wedge \rho Z+\rho Y \wedge Z+\sum_{i=1}^{3} \eta_{i}\left(J_{i} Y \wedge J_{i} Z+2\left\langle J_{i} Y, Z\right\rangle J_{i}\right),
$$

so using (3-15) and the identities

$$
\begin{aligned}
{[a \wedge b, c \wedge d] } & =\langle a, d\rangle c \wedge b-\langle a, c\rangle d \wedge b-\langle b, d\rangle c \wedge a+\langle b, c\rangle d \wedge a, \\
{\left[J_{s}, a \wedge b\right] } & =J_{s} a \wedge b+a \wedge J_{s} b,
\end{aligned}
$$

we obtain

$$
F(Y, Z)=V(Y, Z)+\sum_{i=1}^{3}\left\langle K_{i} Y, Z\right\rangle J_{i}+S(Y, Z)
$$

where

$$
\begin{aligned}
S(Y, Z) & \in(\Phi Y+\Phi Z) \wedge \mathbb{R}^{n}, \\
V(Y, Z) & =-\frac{1}{2} \sum_{i=1}^{3}\left\langle J_{i} Z, Y\right\rangle\left(\lambda_{i}^{2} v \wedge J_{i} v+\varepsilon_{j k}\left(\lambda_{j} \lambda_{k}-\lambda_{i} \lambda_{k}-\lambda_{j} \lambda_{i}\right) J_{j} v \wedge J_{k} v\right) \\
& \in \Phi v \wedge \Phi_{v},
\end{aligned}
$$


and for subspaces $L_{1}, L_{2} \subset \mathbb{R}^{n}$, we denote by $L_{1} \wedge L_{2}$ the subspace of the space $\mathfrak{o}(n)$ of skew-symmetric operators on $\mathbb{R}^{n}$ defined by

$$
L_{1} \wedge L_{2}=\operatorname{Span}\left(a \wedge b: a \in L_{1}, b \in L_{2}\right) .
$$

Note that if $L_{1}$ and $L_{2}$ are $\mathscr{\mathscr { F }}$-invariant (that is, $\mathscr{F} L_{\alpha} \subset L_{\alpha}$ ), then $L_{1} \wedge L_{2}$ is $\operatorname{ad}_{\mathscr{F}-}$ invariant, that is, $\left[J_{s}, L_{1} \wedge L_{2}\right] \subset L_{1} \wedge L_{2}$.

From (3-15) and the facts that

$$
\omega_{i} \lambda_{i}=(24 C)^{-1} \eta_{i}, \quad \frac{d}{d t} \omega_{i}=4 \omega_{i}^{2}+(12 C)^{-1} \eta_{i}, \quad \sum_{i} \omega_{i}^{-1}=0,
$$

which follow from (3-14) and (3-15), we obtain

$$
\begin{aligned}
K_{i}=-\omega_{i}\left(\left(4 \omega_{i}+\lambda_{i}\right) v \wedge J_{i} v+4 \varepsilon_{j k}\left(\omega_{j}+\omega_{k}\right) J_{j} v \wedge J_{k} v\right. & \\
& \left.+\lambda_{i}\left(48 C+\|v\|^{2}\right) J_{i}+\left(J_{i} H+H J_{i}\right)\right),
\end{aligned}
$$

where $\{i, j, k\}=\{1,2,3\}$ and $H$ is the symmetric operator associated to the Hessian of $t$, that is, $\langle H Y, Z\rangle=Y(Z t)-\left(\nabla_{Y} Z\right) t$ for vector fields $Y$ and $Z$ on $U^{\prime}$.

Since $\left[F(Y, Z), J_{s}\right]=0$ and the subspace $\mathscr{I} Y+\mathscr{I} Z$ is $\mathscr{J}$-invariant (and hence

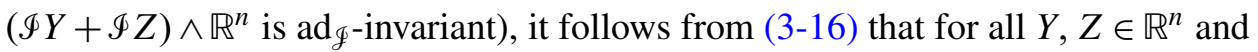
all $s=1,2,3$,

$$
\left[V(Y, Z), J_{s}\right]+\sum_{i=1}^{3}\left\langle K_{i} Y, Z\right\rangle\left[J_{i}, J_{s}\right] \in(\mathscr{Y}+\Phi Z) \wedge \mathbb{R}^{n} .
$$

Take $Y, Z \in \Phi_{v}$ in (3-18). Then $\mathscr{Y}+\Phi_{Z}=\Phi_{v}$ and $\left[V(Y, Z), J_{s}\right] \in \Phi_{v} \wedge I_{v}$ by the $J^{2}$ property, so (3-18) simplifies to $\sum_{i \neq s} \varepsilon_{i s}\left\langle K_{i} Y, Z\right\rangle J_{j} \in I_{v} \wedge \mathbb{R}^{n}$, where $\{i, j, s\}=\{1,2,3\}$. Project this onto the subspace $\left(\Phi_{v}\right)^{\perp} \wedge(\Phi v)^{\perp} \subset \mathfrak{o}(n)$ by the standard inner product on $\mathfrak{o}(n)$, and use that $\left(\Phi_{v}\right)^{\perp}$ is $\mathscr{f}$-invariant and $n \geq 8$. Then we get $\left\langle K_{i} Y, Z\right\rangle=0$ for all $i=1,2,3$ and all $Y, Z \in I_{v}$. Introduce the operators

$$
\hat{J}_{i}=\pi_{\Phi_{v}} J_{i} \pi_{\Phi_{v}} \quad \text { and } \quad \hat{H}=\pi_{\Phi_{v}} H \pi_{\Phi_{v}} \quad \text { on } \Phi_{v} .
$$

Since $\Phi_{v}$ is $\mathscr{g}$-invariant, the $\hat{J}_{i}$ are anticommuting almost Hermitian structures on $I_{v}$. Then the condition $\left\langle K_{i} Y, Z\right\rangle=0$ for $Y, Z \in \Phi_{v}$ and (3-17) imply $\left(4 \omega_{i}+\lambda_{i}\right) v \wedge \hat{J}_{i} v+4 \varepsilon_{j k}\left(\omega_{j}+\omega_{k}\right) \hat{J}_{j} v \wedge \hat{J}_{k} v+\lambda_{i}\left(48 C+\|v\|^{2}\right) \hat{J}_{i}+\hat{J}_{i} \hat{H}+\hat{H} \hat{J}_{i}=0$.

Multiplying by $\hat{J}_{i}$ and taking the trace we obtain for $\{i, j, k\}=\{1,2,3\}$

$$
4\|v\|^{2}\left(\omega_{i}+\omega_{j}+\omega_{k}\right)+\lambda_{i}\left(96 C+3\|v\|^{2}\right)+\operatorname{Tr} \hat{H}=0,
$$

so $\lambda_{i}\left(96 C+3\|v\|^{2}\right)$ does not depend on $i=1,2,3$. Since the $\lambda_{i}$ are pairwise distinct (otherwise the condition $\sum_{i=1}^{3} \eta_{i}^{-1}=0$ from (3-14) is violated), we get $\|v\|^{2}=-32 C$. 
Now take $Y, Z \perp I_{v}$ in (3-18). Projecting to $I_{v} \wedge \Phi_{v}$ and using the fact that $\Phi_{v} \wedge \Phi_{v}$ is adg-invariant we obtain that the operator $V(Y, Z)+\sum_{i=1}^{3}\left\langle K_{i} Y, Z\right\rangle \hat{J}_{i}$ on $\Phi_{v}$ commutes with every $\hat{J}_{s}$. The centralizer of the set $\left\{\hat{J}_{1}, \hat{J}_{2}, \hat{J}_{3}\right\}$ in the Lie algebra $\mathfrak{o}(4)=\mathfrak{o}\left(\Phi_{v}\right)$ is the three-dimensional subalgebra spanned by

$$
v \wedge \hat{J}_{i} v-\varepsilon_{j k} \hat{J}_{j} v \wedge \hat{J}_{k} v \text { for }\{i, j, k\}=\{1,2,3\}
$$

(right multiplication by the imaginary quaternions). Substituting $V(Y, Z)$ from (3-16) and using that

$$
\hat{J}_{i}=\|v\|^{-2}\left(v \wedge \hat{J}_{i} v+\varepsilon_{j k} \hat{J}_{j} v \wedge \hat{J}_{k} v\right),
$$

we obtain that the operator $V(Y, Z)+\sum_{i=1}^{3}\left\langle K_{i} Y, Z\right\rangle \hat{J}_{i}$ commutes with all the $\hat{J}_{s}$ for $Y, Z \perp \Phi_{v}$ if and only if $-\frac{1}{2}\left\langle J_{i} Z, Y\right\rangle\left(\lambda_{i}^{2}+\lambda_{j} \lambda_{k}-\lambda_{i} \lambda_{k}-\lambda_{j} \lambda_{i}\right)+2\|v\|^{-2}\left\langle K_{i} Y, Z\right\rangle=0 \quad$ for all $i=1,2,3$.

Substituting the $\lambda_{i}$ from (3-15) and $\left\langle K_{i} Y, Z\right\rangle$ from (3-17) and taking into account that $\|v\|^{2}=-32 C$, which is shown above, we obtain

$$
\left\langle\left(J_{i} H+H J_{i}-32 C \lambda_{i} J_{i}\right) Y, Z\right\rangle=0 \quad \text { for all } Y, Z \perp \Phi_{v} \text { and all } i=1,2,3 .
$$

Then

$$
\pi\left(J_{i} H+H J_{i}\right) \pi=32 C \lambda_{i} \pi J_{i} \pi,
$$

where $\pi=\pi_{\left(\Phi_{v}\right)^{\perp}}$. Multiplying both sides by $\pi J_{i} \pi$ from the right and using that $\left[\pi, J_{i}\right]=0\left(\right.$ as $\left(\Phi_{v}\right)^{\perp}$ is $\mathscr{f}$-invariant), we get $\pi\left(J_{i} H J_{i}-H\right) \pi=-32 C \lambda_{i} \pi$. Taking the traces of the both sides we obtain $-2 \operatorname{Tr}(\pi H \pi)=-32 C \lambda_{i}(n-4)$, which is a contradiction since $n>4$ and the $\lambda_{i}$ are pairwise distinct, which follows from the equation $\sum_{i=1}^{3} \eta_{i}^{-1}=0$ of (3-14).

The next lemma shows that the relations similar to (3-5) and (3-6) of Lemma 3.5 also hold in all the remaining cases when $n=8$, that is, when $v \neq 3$ and when $v=3$ and $J_{1} J_{2} \neq \pm J_{3}$. As shown in Lemma 3.2, in all these cases the Weyl tensor has a smooth Cliff(7) structure in a neighborhood $U$ of every point $x \in M^{\prime}$. Moreover, Lemma 2.6(2), that Cliff(7) structure is an almost Hermitian octonion structure, in the following sense. For every $y \in \mathcal{U}$, we can identify $\mathbb{R}^{8}=T_{y} M^{8}$ with $\mathbb{O}$ and of $\mathbb{R}^{7}$ with $\mathbb{O}^{\prime}=1^{\perp}$ via linear isometries $l_{1}$ and $\iota_{2}$ respectively so that the orthogonal multiplication (2-2) defined by Cliff(7) has the form (2-5): $J_{u} X=X u$ for every $X \in \mathbb{R}^{8}=\mathbb{O}$ and $u \in \mathbb{O}^{\prime}$.

Lemma 3.6. Let $x \in M^{\prime} \subset M^{8}$, and let $\mathcal{U}$ be the neighborhood of $x$ defined in Lemma 3.2. For every point $y \in U$, identify $\mathbb{R}^{8}=T_{y} M^{8}$ with $\mathbb{O}$ via a linear isometry so that the Clifford structure $\operatorname{Cliff}(7)$ on $\mathbb{R}^{8}$ is given by (2-5). Then there 
exist $m, t, b_{i j} \in \mathbb{R}^{8}=\mathbb{O}$ with $i, j=1, \ldots, 7$ such that for all $X, U \in \mathbb{R}^{8}=\mathbb{O}$,

$$
\begin{aligned}
\left(\nabla_{U} J_{i}\right) X= & \sum_{j=1}^{7}\left\langle b_{i j}, U\right\rangle X e_{j} \\
& +\left(X\left(U^{*} m\right)-\langle m, U\rangle X\right) e_{i}+\left\langle m, U e_{i}\right\rangle X, \\
b_{i j}+b_{j i}= & 0, \\
\left(\nabla_{X} \rho\right) U-\left(\nabla_{U} \rho\right) X= & 2 \sum_{i=1}^{7} \eta_{i}\left(\left\langle m e_{i}, U\right\rangle X e_{i}\right. \\
& +\frac{3}{4}(X \wedge U) t, \\
\nabla \eta_{i}= & -4 \eta_{i} m-\frac{1}{2} t .
\end{aligned}
$$

Proof. In the proof we use standard identities of the octonion arithmetic (some of them are given in Section 2.5).

By [N 2004, Lemma 7], for the Clifford structure Cliff(7) given by (2-5), there exist $b_{i j} \in \mathbb{R}^{8}$, with $i, j=1, \ldots, 7$, satisfying (3-19b) and an (R-)linear operator $A: \mathbb{O} \rightarrow \mathbb{O}^{\prime}$ such that for all $X, U \in \mathbb{R}^{8}=\mathbb{O}$,

$$
\left(\nabla_{U} J_{i}\right) X=\sum_{j=1}^{7}\left\langle b_{i j}, U\right\rangle X e_{j}+(X \cdot A U) e_{i}+\left\langle A U, e_{i}\right\rangle X .
$$

Equation (3-2) is a polynomial equation in 24 real variables, the coordinates of the vectors $X, Y, U \in \mathbb{R}^{8}$. It still holds if we allow $X, Y, U$ to be complex and extend the tensors $J_{i}, \nabla J_{i}$ and $\langle\cdot, \cdot\rangle$ to $\mathbb{C}^{8}$ by complex linearity. The complexified inner product $\langle\cdot, \cdot\rangle$ takes values in $\mathbb{C}$ and is a nonsingular quadratic form on $\mathbb{C}^{8}$. Moreover, Equation (2-5) is still true if we identify $\mathbb{C}^{8}$ with the bioctonion algebra $\mathbb{O} \otimes \mathbb{C}$, and $\mathbb{C}^{7}$ with $1^{\perp}=\mathbb{O}^{\prime} \otimes \mathbb{C}$, the orthogonal complement to 1 in $\mathbb{O} \otimes \mathbb{C}$.

Let $Y \in \mathbb{O} \otimes \mathbb{C}$ be a nonzero isotropic vector (that is, $Y^{*} Y=0$ ) and let

$$
\mathscr{I}_{\mathbb{C}} Y=\operatorname{Span}_{\mathbb{C}}\left(J_{1} Y, \ldots, J_{7} Y\right) .
$$

Then $Y \in \mathscr{S}_{\mathbb{C}} Y$ and the space $\mathscr{E}_{\mathbb{C}} Y$ is isotropic: The inner product of any two vectors from $\mathscr{S}_{\mathbb{C}} Y$ vanishes. Choose $X, U \in \mathscr{E}_{\mathbb{C}} Y$ and take the inner product of the complexified (3-2) with $X$. Since $X, Y$ and $U$ are mutually orthogonal, we get (3-3), which further simplifies to

$$
\sum_{i=1}^{7} \eta_{i}\left\langle J_{i} X, U\right\rangle\left\langle\left(\nabla_{Y} J_{i}\right) Y, X\right\rangle=0
$$

since

$$
\|X\|^{2}=\|Y\|^{2}=\left\langle J_{i} Y, X\right\rangle=\left\langle J_{i} Y, U\right\rangle=0 .
$$


Using (3-20) we obtain

$$
\sum_{i=1}^{7} \eta_{i}\left\langle J_{i} X, U\right\rangle\left\langle(Y \cdot A Y) e_{i}, X\right\rangle=0
$$

for all isotropic vectors $Y$ and for all $X, U \in \mathscr{E}_{\mathbb{C}} Y$. It follows that $Y \cdot A Y$ is perpendicular to $\sum_{i=1}^{7} \eta_{i}\left\langle J_{i} X, U\right\rangle X e_{i}$ for all $X, U \in \mathscr{I}_{\mathbb{C}} Y$. Since $Y \cdot A Y=J_{A Y} Y \in \mathscr{E}_{\mathbb{C}} Y$ and $\mathscr{g}_{\mathbb{C}} Y$ is isotropic, we get $Y \cdot A Y \perp \mathscr{g}_{\mathbb{C}} Y$, so $Y \cdot A Y$ is perpendicular to

$$
\mathscr{I}_{\mathbb{C}} Y+\operatorname{Span}_{\mathbb{C}}\left(\left\{\sum_{i=1}^{7} \eta_{i}\left\langle J_{i} X, U\right\rangle J_{i} X \mid X, U \in \mathscr{g}_{\mathbb{C}} Y\right\}\right) .
$$

Following the arguments in the proof of [N 2004, Lemma 8] starting with formula (29), we obtain that $A U=U^{*} m-\langle U, m\rangle 1$ for some $m \in \mathbb{O}$. Then (3-19a) follows from (3-20).

To prove (3-19c) and (3-19d), introduce the vectors $f_{i j} \in \mathbb{R}^{8}$ for $i, j=1, \ldots, 8$ and the quadratic map $T: \mathbb{R}^{8} \rightarrow \mathbb{R}^{8}$ (similar to the map $Q$ of (3-4)) by

$$
\begin{aligned}
f_{i j} & =\left(\eta_{i}-\eta_{j}\right) b_{i j}+\delta_{i j}\left(\nabla \eta_{i}-2 \eta_{i} m\right), \\
\langle T(X), U\rangle & =\frac{1}{3}\left\langle\left(\nabla_{X} \rho\right) U-\left(\nabla_{U} \rho\right) X, X\right\rangle-\sum_{i=1}^{7} \eta_{i}\left\langle m e_{i}, X\right\rangle\left\langle X e_{i}, U\right\rangle .
\end{aligned}
$$

Note that $f_{i j}=f_{j i}$ and $\langle T(X), X\rangle=0$. Take $X, Y, U$ to be mutually orthogonal vectors in $\mathbb{R}^{8}$. By (3-19a) and (3-19b),

$$
\begin{aligned}
\left\langle\left(\nabla_{U} J_{i}\right) X, Y\right\rangle & =\sum_{j=1}^{7}\left\langle b_{i j}, U\right\rangle\left\langle X e_{j}, Y\right\rangle-\langle m, U\rangle\left\langle X e_{i}, Y\right\rangle+\left\langle\left(X\left(U^{*} m\right)\right) e_{i}, Y\right\rangle \\
& =\sum_{j=1}^{7}\left\langle b_{i j}-\delta_{i j} m, U\right\rangle\left\langle X e_{j}, Y\right\rangle+\left\langle m\left(\left(e_{i} Y^{*}\right) X\right), U\right\rangle,
\end{aligned}
$$

so every term on the left side of (3-3) can be written as the inner product of $U$ with a vector depending on $X$ and $Y$. Since $U$ is arbitrary other than being perpendicular to $X$ and $Y$, we find after substituting (2-5) and (3-19a) into (3-3) and rearranging the terms that

$$
\begin{aligned}
& \|X\|^{2} T(Y)+\|Y\|^{2} T(X) \\
& +\sum_{i=1}^{7}\left(2 \eta_{i}\left\langle Y e_{i}, X\right\rangle\left(m\left(\left(e_{i} Y^{*}\right) X\right)+\left(Y\left(X^{*} m\right)\right) e_{i}\right)\right. \\
& \left.+\left\langle Y e_{j}, X\right\rangle\left(\left\langle f_{i j}, X\right\rangle Y e_{i}-\left\langle f_{i j}, Y\right\rangle X e_{i}\right)-\left\langle Y e_{i}, X\right\rangle\left\langle Y e_{j}, X\right\rangle f_{i j}\right) \\
& \in \operatorname{Span}(X, Y),
\end{aligned}
$$

for all $X \perp Y$, where we used the fact that $\left(X\left(Y^{*} m\right)\right) e_{i}=-\left(Y\left(X^{*} m\right)\right) e_{i}$, since $X \perp Y$. Taking the inner products with $X$ and with $Y$, we obtain that the left side 
of the above (before the " $\in$ ") is equal to $\langle T(Y), X\rangle X+\langle T(X), Y\rangle Y$ for all $X \perp Y$. Taking $X=Y u$ with $u=\sum_{i=1}^{7} u_{i} e_{i} \in \mathbb{O}^{\prime}$ and regrouping the terms, we obtain

$$
\begin{aligned}
& \|u\|^{2} T(Y)+T(Y u) \\
& \quad+2 \sum_{i=1}^{7} \eta_{i} u_{i}\left(2\left\langle Y, m e_{i}\right\rangle Y u-2\left\langle Y u, m e_{i}\right\rangle Y+2\|Y\|^{2}(m u) e_{i}\right) \\
& \quad+\sum_{i, j=1}^{7} u_{j}\left(\left\langle f_{i j}+8 \delta_{i j} \eta_{i} m, Y u\right\rangle Y e_{i}-\left\langle f_{i j}+8 \delta_{i j} \eta_{i} m, Y\right\rangle(Y u) e_{i}\right) \\
& \quad-\sum_{i, j=1}^{7}\|Y\|^{2} u_{i} u_{j} f_{i j}=\|Y\|^{-2}\langle T(Y), Y u\rangle Y u+\|Y\|^{-2}\langle T(Y u), Y\rangle Y,
\end{aligned}
$$

where we used

$m\left(\left(e_{i} Y^{*}\right) X\right)+\left(Y\left(X^{*} m\right)\right) e_{i}$

$=2\left\langle Y, m e_{i}\right\rangle Y u-2\left\langle Y u, m e_{i}\right\rangle Y+4\langle Y u, m\rangle Y e_{i}-4\langle Y, m\rangle(Y u) e_{i}+2\|Y\|^{2}(m u) e_{i}$,

which follows from

$$
m\left(\left(e_{i} Y^{*}\right) X\right)=\left(Y\left(X^{*} m\right)\right) e_{i}-2\left\langle m, Y e_{i}\right\rangle X-2\left\langle X, m e_{i}\right\rangle Y
$$

for all $X, Y$, and

$$
\left(Y\left(X^{*} m\right)\right) e_{i}=-2\langle Y, m\rangle(Y u) e_{i}-2\langle Y, m u\rangle Y e_{i}+\|Y\|^{2}(m u) e_{i}
$$

for $X=Y u$ and $u \perp 1$. By Lemma 2.7(1) (with $v=1$ and $\Phi Y=\operatorname{Span}(Y, Y u)$ ) we obtain that both coefficients on the right side of (3-23), $\|Y\|^{-2}\langle T(Y), Y u\rangle$ and $\|Y\|^{-2}\langle T(Y u), Y\rangle$, are linear forms of $Y \in \mathbb{R}^{8}$ for every $u \in \mathbb{O}^{\prime}$. Since $\langle T(Y), Y\rangle$

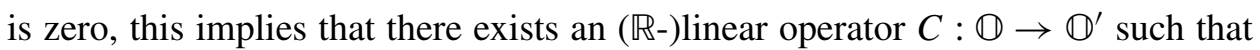
$\|Y\|^{-2} Y^{*} T(Y)=C Y$, so $T(Y)=Y \cdot C Y$ for all $Y \in \mathbb{O}$. Substituting this to (3-23) and rearranging the terms, we obtain

$$
(Y u)\left(C(Y u)-\sum_{i, j=1}^{7} u_{j}\left\langle f_{i j}+8 \delta_{i j} \eta_{i} m, Y\right\rangle e_{i}\right)
$$

$$
\begin{aligned}
+Y\left(\|u\|^{2} C Y+\sum_{i=1}^{7}\left(4 \eta_{i} u_{i}\left(\left\langle Y, m e_{i}\right\rangle u-\left\langle Y u, m e_{i}\right\rangle 1+Y^{*}\left((m u) e_{i}\right)\right)\right.\right. \\
+u_{j}\left\langle f_{i j}+8 \delta_{i j} \eta_{i} m, Y u\right\rangle e_{i} \\
\left.\left.-u_{i} u_{j} Y^{*} f_{i j}-\langle C Y, u\rangle u+\langle C(Y u), u\rangle 1\right)\right)=0
\end{aligned}
$$

The left side of (3-24) has the form $(Y u) L(Y, u)+Y F(Y, u)$, where $L(Y, u)$ and $F(Y, u)$ are $(\mathbb{R}-)$ linear operators on $\mathbb{O}$ for every $u \in \mathbb{O}^{\prime}$. By [N 2004, Lemma 6], for every unit octonion $u \in \mathbb{O}^{\prime}, L(Y, u)=\langle a(u), Y\rangle 1+\langle t(u), Y\rangle u+Y^{*} p(u)$ for 
some functions $a, t, p: S^{6} \subset \mathbb{O}^{\prime} \rightarrow \mathbb{O}$. Extending $a, t, p$ by homogeneity (of degree $1,0,1$ respectively) to $\mathbb{O}^{\prime}$ we obtain

$$
C(Y u)-\sum_{i, j=1}^{7} u_{j}\left\langle f_{i j}+8 \delta_{i j} \eta_{i} m, Y\right\rangle e_{i}=\langle a(u), Y\rangle 1+\langle t(u), Y\rangle u+Y^{*} p(u)
$$

for all $u \in \mathbb{O}^{\prime}$. Moreover, $p(u)=-a(u)$, since $C(Y) \perp 1$. By the linearity of the left side in $u$, we get

$$
\begin{aligned}
\left\langle a\left(u_{1}+u_{2}\right)-\right. & \left.a\left(u_{1}\right)-a\left(u_{2}\right), Y\right\rangle 1+\left\langle t\left(u_{1}+u_{2}\right)-t\left(u_{1}\right), Y\right\rangle u_{1} \\
& +\left\langle t\left(u_{1}+u_{2}\right)-t\left(u_{2}\right), Y\right\rangle u_{2}+Y^{*}\left(a\left(u_{1}+u_{2}\right)-a\left(u_{1}\right)-a\left(u_{2}\right)\right)=0
\end{aligned}
$$

for all $u_{1}, u_{2} \in \mathbb{O}^{\prime}$. Then $Y^{*}\left(a\left(u_{1}+u_{2}\right)-a\left(u_{1}\right)-a\left(u_{2}\right)\right) \in \operatorname{Span}\left(1, u_{1}, u_{2}\right)$ for all $Y \in \mathbb{O}$, which is only possible when $a(u)$ is linear, that is, $a(u)=B u$ for some $\left(\mathbb{R}\right.$-)linear operator $B: \mathbb{O}^{\prime} \rightarrow \mathbb{O}$. It follows that $t\left(u_{1}+u_{2}\right)=t\left(u_{1}\right)=t\left(u_{2}\right)$, that is, $t \in \mathbb{O}$ is a constant. So

$$
C(Y u)=\sum_{i, j=1}^{7} u_{j}\left\langle f_{i j}+8 \delta_{i j} \eta_{i} m, Y\right\rangle e_{i}+\langle B u, Y\rangle 1+\langle t, Y\rangle u-Y^{*} B u .
$$

Taking the inner product of the both sides with $v \in \mathbb{O}^{\prime}$ and subtracting from the resulting equation the same equation with $u$ and $v$ interchanged, we obtain $\langle C(Y u), v\rangle-\langle C(Y v), u\rangle=\langle B v, Y u\rangle-\langle B u, Y v\rangle$, since $f_{i j}=f_{j i}$ by (3-21). It follows that $\left\langle C^{t} v-B v, Y u\right\rangle=\left\langle C^{t} u-B u, Y v\right\rangle$, where $C^{t}$ is the operator adjoint to $C$. Now taking $u \perp v$ and $Y=u v$, we get

$$
\|u\|^{2}\left\langle C^{t} v-B v, v\right\rangle=-\|v\|^{2}\left\langle C^{t} u-B u, u\right\rangle,
$$

which implies $C=B^{t}$. Then from the above,

$$
\left\langle C(Y u), e_{i}\right\rangle=\sum_{j=1}^{7} u_{j}\left\langle f_{i j}+8 \delta_{i j} \eta_{i} m, Y\right\rangle+\langle t, Y\rangle u_{i}-\left\langle B u, Y e_{i}\right\rangle=\left\langle B e_{i}, Y u\right\rangle,
$$

so $\sum_{j=1}^{7} u_{j}\left(f_{i j}+\delta_{i j}\left(8 \eta_{i} m+t\right)\right)+(B u) e_{i}+\left(B e_{i}\right) u=0$. Therefore

(3-25) $T(Y)=Y \cdot C Y=Y \cdot B^{t} Y \quad$ and $\quad f_{i j}=-\delta_{i j}\left(8 \eta_{i} m+t\right)-\left(B e_{i}\right) e_{j}-\left(B e_{j}\right) e_{i}$.

Substituting (3-25) to (3-24) and simplifying, we obtain

$$
-\langle L u \cdot u, Y\rangle Y-\langle L u, Y\rangle Y u+\|Y\|^{2} L u \cdot u=0,
$$

where $L u=4 B u-t u-4 \sum_{i=1}^{7} \eta_{i} u_{i} m e_{i}$. Taking $Y \perp L u, L u \cdot u$ we get $L u=0$, so

$$
B u=\frac{1}{4} t u+\sum_{i=1}^{7} \eta_{i} u_{i} m e_{i} .
$$


Substituting (3-26) into the first equation of (3-25) and then into (3-22) and simplifying, we obtain that for arbitrary $X, U \in \mathbb{O}$,

$$
\begin{aligned}
& \left\langle\left(\nabla_{X} \rho\right) U-\left(\nabla_{U} \rho\right) X, X\right\rangle \\
& \quad=\frac{3}{4}\left(\langle t, X\rangle\langle X, U\rangle-\|X\|^{2}\langle t, U\rangle\right)+6 \sum_{i=1}^{7} \eta_{i}\left\langle X e_{i}, U\right\rangle\left\langle m e_{i}, X\right\rangle .
\end{aligned}
$$

Polarizing this equation we get

$$
\begin{aligned}
& \left\langle\left(\nabla_{Y} \rho\right) U-\left(\nabla_{U} \rho\right) Y, X\right\rangle+\left\langle\left(\nabla_{X} \rho\right) U-\left(\nabla_{U} \rho\right) X, Y\right\rangle \\
& =\frac{3}{4}(\langle t, X\rangle\langle Y, U\rangle+\langle t, Y\rangle\langle X, U\rangle-2\langle X, Y\rangle\langle t, U\rangle) \\
& \quad+6 \sum_{i=1}^{7} \eta_{i}\left(\left\langle X e_{i}, U\right\rangle\left\langle m e_{i}, Y\right\rangle+\left\langle Y e_{i}, U\right\rangle\left\langle m e_{i}, X\right\rangle\right) .
\end{aligned}
$$

Subtracting the same equation with $X$ and $U$ interchanged and using the fact that $\rho$ is symmetric, we get (3-19c). The second equation of (3-25) and (3-26) give $f_{i i}=-6 \eta_{i} m-t / 2$, which by (3-21) implies (3-19d).

Lemma 3.7. In the assumptions of Theorem 3.1, let $x \in M^{\prime}$, where $M^{\prime} \subset M^{n}$ is defined in Lemma 3.2. Then there exists a neighborhood $U=U(x)$ and a smooth metric on U conformally equivalent to the original metric whose curvature tensor has the form (3-1), with $\rho$ a multiple of the identity.

Proof. Let $x \in M^{\prime}$ and let $U$ be the neighborhood of $x$ on which the Weyl tensor has the smooth Clifford structure defined in Lemma 3.2. We can assume that $v>0$, since in the case of a $\operatorname{Cliff}(0)$ structure, the curvature tensor given by (3-1) has the form $R(X, Y) Z=\langle X, Z\rangle \rho Y+\langle\rho X, Z\rangle Y-\langle Y, Z\rangle \rho X-\langle\rho Y, Z\rangle X$, so the Weyl tensor vanishes. Then the metric on $U$ is locally conformally flat, that is, is conformally equivalent to a one with $\rho=0$.

If $n=8$ and $v=7$, and all the $\eta_{i}$ at $x$ are equal, then they are equal at some neighborhood of $x$ (by the definition of $M^{\prime}$ ). By Remark 3.4, we can replace $\rho$ by $\rho+3 \eta_{1} / 2$ id and $\eta_{i}$ by $0=\eta_{i}-\eta_{1}$ in (3-1), thus arriving at the case $v=0$ considered above.

For the remaining part of the proof, we will assume that in the case $n=8$ and $v=7$, at least two of the $\eta_{i}$ at $x$ are different; up to relabeling, let $\eta_{1} \neq \eta_{2}$ at $x$ and also on a neighborhood of $x$ (replace $U$ by a smaller neighborhood if necessary). Let $f$ be a smooth function on $U$ and let $\langle\cdot, \cdot\rangle^{\prime}=e^{f}\langle\cdot, \cdot\rangle$. Then $W^{\prime}=W, J_{i}^{\prime}=J_{i}$, $\eta_{i}^{\prime}=e^{-f} \eta_{i}$ and on functions, $\nabla^{\prime}=e^{-f} \nabla$, where we use the prime for objects associated to the metric $\langle\cdot, \cdot\rangle^{\prime}$. Moreover, the curvature tensor $R^{\prime}$ still has the form (3-1), and all the identities of Lemmas 3.5 and 3.6 remain valid.

In the cases considered in Lemma 3.5, the ratios $\eta_{i} / \eta_{1}$ are constant, which follows from (3-5d) and (3-6c). In particular, taking $f=\ln \left|\eta_{1}\right|$ we obtain that $\eta_{1}^{\prime}$ is a constant, so all the $\eta_{i}^{\prime}$ are constant; $m_{i}^{\prime}=0$ by $(3-5 \mathrm{~d})$, so $\left(\nabla_{Y}^{\prime} \rho^{\prime}\right) U-\left(\nabla_{U}^{\prime} \rho^{\prime}\right) Y=0$ 
by (3-6a). In the case $n=8$ and $v=7$ (Lemma 3.6), take $f=\ln \left|\eta_{1}-\eta_{2}\right|$. Then by (3-19d), we have $\nabla f=-4 m$ and $\nabla^{\prime} \eta_{i}^{\prime}=-(1 / 2) e^{-2 f} t$, which imply $m^{\prime}=-(1 / 4) \nabla^{\prime} \ln \left|\eta_{1}^{\prime}-\eta_{2}^{\prime}\right|=0$ and $t^{\prime}=e^{-2 f} t$, again by (3-19d) for the metric $\langle\cdot, \cdot\rangle^{\prime}$. Then by (3-19c), we have $\left(\nabla_{X}^{\prime} \rho^{\prime}\right) U-\left(\nabla_{U}^{\prime} \rho^{\prime}\right) X=(3 / 4)\left(X \wedge^{\prime} U\right) t^{\prime}$. By Remark 3.4, we can replace $\rho^{\prime}$ by $\tilde{\rho}=\rho^{\prime}+(3 / 2)\left(\eta_{1}^{\prime}+C\right)$ id and $\eta_{i}^{\prime}$ by $\tilde{\eta}_{i}=\eta_{i}^{\prime}-\left(\eta_{1}^{\prime}+C\right)$ without changing the curvature tensor $R^{\prime}$ given by (3-1). (Here $C$ is a constant chosen in such a way that $\tilde{\eta}_{i} \neq 0$ anywhere on $\mathcal{U}$.) Then by (3-19c) and (3-19d), $\left(\nabla_{X}^{\prime} \tilde{\rho}\right) U-\left(\nabla_{U}^{\prime} \tilde{\rho}\right) X=0$ for the metric $\langle\cdot, \cdot\rangle^{\prime}$.

Dropping the primes and the tildes, we obtain that, up to a conformal smooth change of the metric on $u$, the curvature tensor has the form (3-1), with $\rho$ satisfying the identity

$$
\left(\nabla_{Y} \rho\right) X=\left(\nabla_{X} \rho\right) Y \text { for all } X, Y,
$$

that is, with $\rho$ being a symmetric Codazzi tensor.

Then by [Derdziński and Shen 1983, Theorem 1], at every point of $u$ for any three eigenspaces $E_{\beta}, E_{\gamma}, E_{\alpha}$ of $\rho$ with $\alpha \notin\{\beta, \gamma\}$, the curvature tensor satisfies $R(X, Y) Z=0$ for all $X \in E_{\beta}, Y \in E_{\gamma}$ and $Z \in E_{\alpha}$. It then follows from (3-1) that

$$
\sum_{i=1}^{\nu} \eta_{i}\left(2\left\langle J_{i} X, Y\right\rangle J_{i} Z+\left\langle J_{i} Z, Y\right\rangle J_{i} X-\left\langle J_{i} Z, X\right\rangle J_{i} Y\right)=0
$$

for all $X \in E_{\beta}, Y \in E_{\gamma}, Z \in E_{\alpha}$, with $\alpha \notin\{\beta, \gamma\}$.

Suppose $\rho$ is not a multiple of the identity. Let $E_{1}, \ldots, E_{p}$ for $p \geq 2$ be the eigenspaces of $\rho$. If $p>2$, write $E_{1}^{\prime}=E_{1}$ and $E_{2}^{\prime}=E_{2} \oplus \cdots \oplus E_{p}$. Then by linearity, (3-27) holds for any $X, Y \in E_{\alpha}^{\prime}$ and $Z \in E_{\beta}^{\prime}$ such that $\{\alpha, \beta\}=\{1,2\}$. Hence to prove the lemma it suffices to show that (3-27) leads to a contradiction in the assumption $p=2$. For the rest of the proof, suppose that $p=2$. Let $d_{\alpha}=\operatorname{dim} E_{\alpha}$ with $d_{1} \leq d_{2}$.

Choose $Z \in E_{\alpha}, \quad X, Y \in E_{\beta}, \quad \alpha \neq \beta$, and take the inner product of (3-27) with $X$. We get $\sum_{i=1}^{v} \eta_{i}\left\langle J_{i} X, Y\right\rangle\left\langle J_{i} X, Z\right\rangle=0$. It follows that for every $X \in$ $E_{\alpha}$, the subspaces $E_{1}$ and $E_{2}$ are invariant subspaces of the symmetric operator $\hat{R}_{X} \in \operatorname{End}\left(\mathbb{R}^{n}\right)$ defined by $\hat{R}_{X} Y=\sum_{i=1}^{v} \eta_{i}\left\langle J_{i} X, Y\right\rangle J_{i} X$. So $\hat{R}_{X}$ commutes with the orthogonal projections $\pi_{\beta}: \mathbb{R}^{n} \rightarrow E_{\beta}$ for $\beta=1,2$. Then for all $\alpha, \beta=1,2$ ( $\alpha$ and $\beta$ can be equal), all $X \in E_{\alpha}$ and all $Y \in \mathbb{R}^{n}$, we have

$$
\sum_{i=1}^{\nu} \eta_{i}\left\langle J_{i} X, \pi_{\beta} Y\right\rangle J_{i} X=\sum_{i=1}^{\nu} \eta_{i}\left\langle J_{i} X, Y\right\rangle \pi_{\beta} J_{i} X .
$$

Taking $Y=J_{j} X$ we get that $\pi_{\beta} J_{j} X \subset \mathscr{E} X$; that is, $\pi_{\beta} \mathscr{\mathscr { I }} \subset \subset \mathscr{\mathscr { J }} X$ for all $X \in E_{\alpha}$

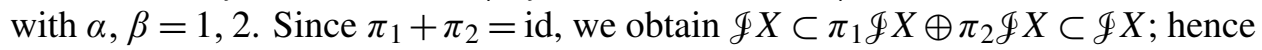
$\mathscr{g} X=\pi_{1} \mathscr{\mathscr { X }} \oplus \pi_{2} \mathscr{\mathscr { I }} X$. Since every function $f_{\alpha \beta}: E_{\alpha} \rightarrow \mathbb{Z}, X \mapsto \operatorname{dim} \pi_{\beta} \mathscr{\mathscr { F }} X$ with $\alpha, \beta=1,2$ is lower semicontinuous, and $f_{\alpha 1}(X)+f_{\alpha 2}(X)=v$ for all nonzero 
$X \in E_{\alpha}$, there exist constants $c_{\alpha \beta}$ with $c_{\alpha 1}+c_{\alpha 2}=v$ such that $\operatorname{dim} \pi_{\beta} \mathscr{\mathscr { I }} X=c_{\alpha \beta}$ for all $\alpha, \beta=1,2$ and all nonzero $X \in E_{\alpha}$.

Let $X, Y \in E_{\alpha}, Z \in E_{\beta}$ and $\beta \neq \alpha$. Taking the inner product of (3-27) with $J_{j} Z$ for $j=1, \ldots, v$, we get

$$
2 \eta_{j}\left\langle J_{j} X, Y\right\rangle\|Z\|^{2}=\sum_{i \neq j} \eta_{i}\left(\left\langle J_{i} Z, X\right\rangle\left\langle J_{i} Y, J_{j} Z\right\rangle-\left\langle J_{i} Z, Y\right\rangle\left\langle J_{i} X, J_{j} Z\right\rangle\right) .
$$

Since $\left\langle J_{i} Z, X\right\rangle=\left\langle J_{i} \pi_{\beta} Z, X\right\rangle=-\left\langle Z, \pi_{\beta} J_{i} X\right\rangle$ (and similarly for $\left\langle J_{i} Z, Y\right\rangle$ ), the right side, viewed as a quadratic form of $Z \in E_{\beta}$, vanishes for all $Z$ in the intersection of $\left.\pi_{\beta} \mathscr{I} X\right)^{\perp}$ and $\left(\pi_{\beta} \mathscr{Y} Y\right)^{\perp}$, that is, on a subspace of dimension at least $d_{\beta}-2 c_{\alpha \beta}$. So for $\alpha \neq \beta$, either $2 c_{\alpha \beta} \geq d_{\beta}$, or $\mathscr{E} E_{\alpha} \perp E_{\alpha}$, that is, $\pi_{\beta} \mathscr{E} X=\mathscr{F} X$ for all $X \in E_{\alpha}$, so $c_{\alpha \beta}=v$.

Similarly, if $Z \in E_{\alpha}, \quad X, Y \in E_{\beta}$ and $\beta \neq \alpha$, the inner product of (3-27) with $J_{j} X$ for $j=1, \ldots, v$ gives

Because

$$
\eta_{j}\left\langle J_{j} Z, Y\right\rangle\|X\|^{2}=\sum_{i=1}^{v} \eta_{i}\left(-2\left\langle J_{i} X, Y\right\rangle\left\langle J_{i} Z, J_{j} X\right\rangle+\left\langle J_{i} Z, X\right\rangle\left\langle J_{i} Y, J_{j} X\right\rangle\right) .
$$

$$
\left\langle J_{i} X, Y\right\rangle=-\left\langle X, \pi_{\beta} J_{i} Y\right\rangle \quad \text { and } \quad\left\langle J_{i} Z, X\right\rangle=-\left\langle X, \pi_{\beta} J_{i} Z\right\rangle,
$$

the right side, viewed as a quadratic form of $X \in E_{\beta}$, vanishes on the intersection of $\left(\pi_{\beta} \mathscr{I} Y\right)^{\perp}$ and $\left(\pi_{\beta} \mathscr{I} Z\right)^{\perp}$, whose dimension is at least $d_{\beta}-c_{\alpha \beta}-c_{\beta \beta}$. We obtain that for $\alpha \neq \beta$, either $c_{\alpha \beta}+c_{\beta \beta} \geq d_{\beta}$, or $\mathscr{E} E_{\alpha} \perp E_{\beta}$, that is, $\pi_{\beta} \mathscr{E} Z=0$ for all $Z \in E_{\alpha}$, so $c_{\alpha \beta}=0$. Since $c_{\alpha \beta}=0$ contradicts both $2 c_{\alpha \beta} \geq d_{\beta}$ and $c_{\alpha \beta}=v$ (since $v>0$ ), we must have $c_{\alpha \beta}+c_{\beta \beta} \geq d_{\beta}$. Then $2 v=\sum_{\alpha \beta} c_{\alpha \beta} \geq d_{1}+d_{2}=n$.

This proves the lemma in all the cases when $2 v<n$, that is, in all the cases except for $n=8$ and $v \geq 4$ (which follows from Lemma 2.4).

Consider the case $n=8$. We identify $\mathbb{R}^{8}$ with $\mathbb{O}$ and assume that the $J_{i}$ act as in (2-5). Let $D: \mathbb{O} \rightarrow \mathbb{O}$ be the symmetric operator defined by $D 1=0$ and $D e_{i}=\eta_{i} e_{i}$. By (2-4), condition (3-27) still holds if we replace $D$ by $D+c \operatorname{Im}$, where $\mathrm{Im}$ is the operator of taking the imaginary part of an octonion. So we can assume that the eigenvalue of the maximal multiplicity of $\left.D\right|_{\mathbb{O}^{\prime}}$ is zero (one of them, if there are more than one). Then in (3-27), $v=\operatorname{rk} D$. By construction, we have $v \leq 6$, and we only need to consider the cases when $v \geq 4$, as shown above.

By $(2-5)$,

$$
\left\langle J_{i} X, Y\right\rangle J_{i} Z=\left\langle X e_{i}, Y\right\rangle Z e_{i}=\left\langle e_{i}, X^{*} Y\right\rangle Z e_{i},
$$

SO

$$
\sum_{i=1}^{v} \eta_{i}\left\langle J_{i} X, Y\right\rangle J_{i} Z=\sum_{i=1}^{v} \eta_{i}\left\langle e_{i}, X^{*} Y\right\rangle Z e_{i}=\sum_{i=1}^{7}\left\langle D e_{i}, X^{*} Y\right\rangle Z e_{i}=Z D\left(X^{*} Y\right),
$$


since $D$ is symmetric and $D 1=0$. Then (3-27) can be rewritten as

$$
2 Z D\left(X^{*} Y\right)+X D\left(Z^{*} Y\right)-Y D\left(Z^{*} X\right)=0
$$

for all $X, Y \in E_{\beta}, Z \in E_{\alpha}$ an $\alpha \neq \beta$.

Taking the inner product of (3-28) with $X$ (and using the fact that $D$ is symmetric, $D 1=0$ and $\left.Y^{*} X=2\langle X, Y\rangle 1-X^{*} Y\right)$, we obtain $\left\langle D\left(X^{*} Y\right), X^{*} Z\right\rangle=0$. It follows that for every $X \in E_{\beta}$, the subspaces $E_{1}$ and $E_{2}$ are invariant subspaces of the symmetric operator $L_{X} D L_{X}^{t}$, where $L_{X}: \mathbb{O} \rightarrow \mathbb{O}$ is the left multiplication by $X$ (note that $L_{X^{*}}=L_{X}^{t}$ and that $L_{X} D L_{X}^{t}$ coincides with the operator $\hat{R}_{X}$ introduced above). So $L_{X} D L_{X}^{t}$ commutes with both orthogonal projections $\pi_{\alpha}: \mathbb{R}^{8} \rightarrow E_{\alpha}$ for $\alpha=1$, 2. It follows that for every $\alpha, \beta$ (not necessarily distinct) and every $X \in E_{\beta}$, the operator $D$ commutes with $L_{X}^{t} \pi_{\alpha} L_{X}=\|X\|^{2} \pi_{X^{*} E_{\alpha}}$, that is,

$$
X^{*} E_{\alpha} \text { is an invariant subspace of } D \text { for all } \alpha, \beta \text {, and all } X \in E_{\beta} \text {. }
$$

Consider all the possible cases for the dimensions $d_{\alpha}$ of the subspaces $E_{\alpha}$.

Let $\left(d_{1}, d_{2}\right)=(1,7)$, and let $u$ be a nonzero vector in $E_{1}$. Then by (3-29), every line spanned by $X^{*} u$ with $X \perp u$ (that is, every line in $\mathbb{O}^{\prime}$ ) is an invariant subspace of $D$. It follows that $\left.D\right|_{\mathbb{O}^{\prime}}$ is a multiple of the identity, which is a contradiction since $\operatorname{rk} D=v$ for $4 \leq v \leq 6$.

Let $\left(d_{1}, d_{2}\right)=(2,6)$, and let $E_{1}=\operatorname{Span}(u, u e)$ for $e \in \mathbb{O}^{\prime}$, and let $\|e\|=\|u\|=1$. Then $E_{2}=u L$, where $L=\operatorname{Span}(1, e)^{\perp}$. Let $U$ be any element of $L$. By (3-29) with $E_{\alpha}=E_{1}$ and $X=u U^{*}=-u U \in E_{2}$, every two-plane $\operatorname{Span}\left(U,\left(U u^{*}\right)(u e)\right)$ is an invariant subspace of $D$. Note that $\left(U u^{*}\right)(u e) \in L$, and that the operator $J$ defined by $J U=\left(U u^{*}\right)(u e)$ is an almost Hermitian structure on $L$. Then $L$ is an invariant subspace of $D$ since it is as the sum of invariant subspaces $\operatorname{Span}(U, J U)$ and $\left.J D\right|_{L} U \in \operatorname{Span}(U, J U)$ (since $\operatorname{Span}(U, J U)$ is both $J$ - and $\left.D\right|_{L}$-invariant). From Lemma 2.7(1), it follows that the operator $\left.J D\right|_{L}$ is a linear combination of $\left.\mathrm{id}\right|_{L}$ and $J$. Since $D$ is symmetric and its eigenvalue of maximal multiplicity is zero, we have $\left.D\right|_{L}=0$. Then $v=\operatorname{rk} D \leq 1$, which is a contradiction.

For the cases $\left(d_{1}, d_{2}\right)=(3,5),(4,4)$, we use the notion of Cayley plane. A four-dimensional subspace $\mathscr{C} \subset \mathbb{O}$ is called a Cayley plane if $X\left(Y^{*} Z\right) \in \mathscr{C}$ for orthonormal octonions $X, Y, Z \in \mathscr{C}$. This definition coincides with [Harvey and Lawson 1982, Definition IV.1.23], if we disregard the orientation. We will need the following properties of the Cayley plane (they can be found in [ibid., Section IV] or proved directly):

(i) A Cayley plane is well defined; moreover, if $X\left(Y^{*} Z\right) \in \mathscr{C}$ for some triple $X, Y, Z$ of orthonormal octonions in $\mathscr{C}$, then the same is true for any (possibly nonorthonormal) triple $X, Y, Z \in \mathscr{C}$. 
(ii) If $\mathscr{C}$ is a Cayley plane, then the subspace $X^{*} \mathscr{C}$ is the same for all nonzero $X \in \mathscr{C}$; we call this subspace $\mathscr{C}^{*} \mathscr{b}$.

(iii) If $\mathscr{C}$ is a Cayley plane, then $\mathscr{C}^{\perp}$ is also a Cayley plane and $\mathscr{C}^{\perp *} \mathscr{C}^{\perp}=\mathscr{C}^{*} \mathscr{b}$. Moreover, for all nonzero $X \in \mathscr{C}^{\perp}$, the subspace $X^{*} \mathscr{C}$ is the same and is equal to $(\mathscr{b} * \mathscr{C})^{\perp}$.

(iv) For every nonzero $e \in \mathbb{O}$ and every pair of orthonormal imaginary octonions $u, v$, the subspace $\mathscr{C}=\operatorname{Span}(e, e u, e v,(e u) v)$ is a Cayley plane; every Cayley plane can be obtained in this way.

Let $\left(d_{1}, d_{2}\right)=(3,5)$. Then $E_{1}$ is contained in a Cayley plane $\mathscr{C}$ (spanned by $E_{1}$ and $X\left(Y^{*} Z\right)$ for some orthonormal vectors $\left.X, Y, Z \in E_{1}\right)$, so $\mathscr{C}^{\perp} \subset E_{2}$. Let $U$ be a unit vector in the orthogonal complement to $\mathscr{C}^{\perp}$ in $E_{2}$. Then $X^{*} E_{2}=\mathscr{C}^{*} \mathscr{C} \oplus$ $\mathbb{R}\left(X^{*} U\right)$ for every nonzero $X \in \mathscr{C}^{\perp}$ by properties (ii) and (iii). Since for any two invariant subspaces of a symmetric operator, their intersection and the orthogonal complement to it in each of them are also invariant, it follows from (3-29) that both $\mathscr{C} * \mathscr{C}$ and every line $\mathbb{R}\left(X^{*} U\right)$ with $X \in \mathscr{C}^{\perp}$ are invariant subspaces of $D$. Then $D$ restricts to a multiple of the identity on the four-dimensional space $\left(\mathscr{C}^{\perp}\right)^{*} U$. Since the eigenvalue of maximal multiplicity of $D$ is zero, $\mathbb{R} 1 \oplus\left(\mathscr{C}^{\perp}\right)^{*} U \subset \operatorname{Ker} D$. Then $v=\operatorname{rk} D \leq 3$, which is again a contradiction.

Let now $d_{1}=d_{2}=4$. First suppose $E_{1}$ is not a Cayley plane. Let $X_{1}$ and $X_{2}$ be orthonormal vectors in $E_{1}$. Then $X_{1}^{*} E_{1} \cap X_{2}^{*} E_{1}$ contains $\operatorname{Span}\left(1, X_{1}^{*} X_{2}\right)$, since $X_{2}^{*} X_{1}=-X_{1}^{*} X_{2}$. Also, for any unit vector $Y \in X_{1}^{*} E_{1} \cap X_{2}^{*} E_{1}$ orthogonal to $\operatorname{Span}\left(1, X_{1}^{*} X_{2}\right)$, we have $Y=X_{1}^{*} X_{3}=X_{2}^{*} X_{4}$ for some $X_{3}, X_{4} \in E_{1}$ such that $X_{3}, X_{4} \perp X_{1}, X_{2}$, which implies $X_{2}\left(X_{1}^{*} X_{3}\right)=X_{4} \in E_{1}$, so $E_{1}$ is a Cayley plane by property (i). It follows that $X_{1}^{*} E_{1} \cap X_{2}^{*} E_{1}=\operatorname{Span}\left(1, X_{1}^{*} X_{2}\right)$. Since by (3-29) both subspaces on the left side are invariant under $D$ and since $\mathbb{R} 1$ is an invariant subspace of $D$, we obtain that every line $\mathbb{R}\left(X_{1}^{*} X_{2}\right)$ for $X_{1}, X_{2} \in E_{1}$ is an invariant subspace of $D$ (that is, $X_{1}^{*} X_{2}$ is an eigenvector of $D$ ). Then the space $L=\operatorname{Span}\left(E_{1}^{*} E_{1}\right)$ lies in an eigenspace of $D$, so $\left.D\right|_{L}$ is a multiple of id $\left.\right|_{L}$. If $X_{1}, X_{2}, X_{3} \in E_{1}$ are orthonormal, then $X_{2}^{*} X_{3} \notin X_{1}^{*} E_{1}$, since $E_{1}$ is not a Cayley plane. So $\operatorname{dim} L \geq 5$. Since the eigenvalue of maximal multiplicity of $D$ is zero, $v=\operatorname{rk} D \leq 3$, a contradiction.

Let again $d_{1}=d_{2}=4$, and let $E_{1}$ be a Cayley plane. Then $E_{2}=\left(E_{1}\right)^{\perp}$ is also a Cayley plane by property (iii). Also, by the same property, $E_{1}^{*} E_{1}=E_{2}^{*} E_{2}=V_{1}$ and $E_{1}^{*} E_{2}=E_{1}^{*} E_{2}=V_{2}$, where $V_{1}$ and $V_{2}$ are mutually orthogonal four-dimensional subspaces of $\mathbb{O}$, and $1 \in V_{1}$. From (3-29), both $V_{1}$ and $V_{2}$ are invariant under $D$. Let $X, Y \in E_{1}$ and $Z, W \in E_{2}$, with $X, Z \neq 0$, and let $u=X^{-1} Y$ and $v=Z^{-1} W$. Since $X^{-1}=\|X\|^{-2} X^{*}$, we have $L_{X^{-1}} E_{1}=V_{1}$ by property (ii). Similarly, $L_{Z^{-1}} E_{2}=V_{1}$. Taking the inner product of (3-28) with $W$ we obtain

$$
2\|Z\|^{2}\|X\|^{2}\langle D u, v\rangle-\left\langle D\left(Z^{*}(X u)\right), Z^{*}(X v)\right\rangle=-\left\langle D\left(Z^{*} X\right), Z^{*}((X u) v)\right\rangle
$$


for all $X \in E_{1}, Z \in E_{2}$ and $u, v \in V_{1}$. The left side is symmetric in $u, v$. Since $(X u) v=-(X v) u$ for any $u \perp v$ with $u, v \perp 1$, we obtain $\left\langle D\left(Z^{*} X\right), Z^{*}((X u) v)\right\rangle=0$ for all $u, v \in V_{1}$ with $u \perp v$ and $u, v \perp 1$, and all $X \in E_{1}$ and $Z \in E_{2}$. Given any nonzero orthogonal $X, X^{\prime} \in E_{1}$, we can find $u, v \in V_{1}$ with $u \perp v$ and $u, v \perp 1$ such that $X^{\prime}=(X u) v$. To see this, note that $X u \in E_{1}$ for every $u \in V_{1}=E_{1}^{*} E_{1}$ by property (i). Since $L_{X}$ is nonsingular, $L_{X}\left(V_{1} \cap 1^{\perp}\right)$ is a three-dimensional subspace of $E_{1}$. The same is true with $X$ replaced by $X^{\prime}$. Therefore $X u=X^{\prime} v$ for some $u, v \in V_{1} \cap 1^{\perp}$; hence $X^{\prime}=-\|v\|^{-2}(X u) v$. Since $X^{\prime} \perp X$, we get $\langle X,(X u) v\rangle=0$, so $u \perp v$. Thus $\left\langle D\left(Z^{*} X\right), Z^{*} X^{\prime}\right\rangle=0$ for any $Z \in E_{2}$ and any orthogonal $X, X^{\prime} \in E_{1}$. Since $Z^{*} E_{1}=V_{2}$ for any nonzero $Z \in E_{2}$ by properties (ii) and (ii), and since the operator $L_{Z^{*}}$ is orthogonal when $\|Z\|=1$, we get $\left\langle D v_{1}, v_{2}\right\rangle=0$ for any two orthogonal vectors $v_{1}, v_{2} \in V_{2}$. It follows that the restriction of $D$ to its invariant subspace $V_{2}$ is a multiple of the identity. Since $V_{2} \subset \mathbb{O}^{\prime}$ and the eigenvalue of $\left.D\right|_{\mathbb{O}^{\prime}}$ of maximal multiplicity is zero, we obtain $\mathbb{R} 1 \oplus V_{2} \subset \operatorname{Ker} D$. Then $v=\operatorname{rk} D \leq 3$, which is a contradiction.

Remark 3.8. It follows from the proof of Lemma 3.7 that the algebraic statement "a symmetric operator satisfying (3-27) is a multiple of the identity" is valid when $2 v<n$. In particular, when $n=16$, it remains true if we relax the restrictions $v \leq 4$ of Theorem 3.1 to $v \neq 8$ (as for $n=16$ and $v \leq 8$ by (2-3)).

Lemma 3.7 implies Theorem 3.1 at the generic points. Indeed, by Lemma 3.7, every $x \in M^{\prime}$ has a neighborhood $U$ that is either conformally flat or is conformally equivalent to a Riemannian manifold whose curvature tensor has the form (3-1), with $\rho$ being a multiple of the identity, that is, whose curvature tensor has a Clifford structure. It follows from [N 2003, Theorem 1.2] and [N 2004, Proposition 2] that $\mathcal{U}$ is conformally equivalent to an open subset of one of five model spaces: the rank-one symmetric spaces $\mathbb{C} P^{n / 2}, \mathbb{C} H^{n / 2}, \mathbb{H} P^{n / 4}$ or $\mathbb{H} H^{n / 4}$, or Euclidean space.

To prove Theorem 3.1 in full, we show first that the same is true for any $x \in M^{n}$, and second that the model space, to a domain of which $U$ is conformally equivalent, is the same for all $x \in M^{n}$.

We normalize the standard metric $\tilde{g}$ on each of the spaces $\mathbb{C} P^{n / 2}, \mathbb{C} H^{n / 2}, \mathbb{H} P^{n / 4}$ and $\mathbb{H} H^{n / 4}$ so that the sectional curvature $K_{\sigma}$ satisfies $\left|K_{\sigma}\right| \in[1,4]$. Then the curvature tensor of each has a Clifford structure $\operatorname{Cliff}\left(\nu ; J_{1}, \ldots, J_{v} ; \varepsilon, \varepsilon, \ldots, \varepsilon\right)$. Here $v=1,3$ and $\varepsilon= \pm 1$. The $J_{i}$ are smooth anticommuting almost Hermitian structures with $J_{1} J_{2}= \pm J_{3}$ when $v=3$, and satisfy

$$
\tilde{\nabla}_{Z} J_{i}=\sum_{j=1}^{m} \omega_{i}^{j}(Z) J_{j},
$$

where $\omega_{i}^{j}$ are smooth 1-forms with $\omega_{i}^{j}+\omega_{j}^{i}=0$ and $\tilde{\nabla}$ is the Levi-Civita connection for $\tilde{g}$. Denote the corresponding spaces by $M_{\nu, \varepsilon}$ and their Weyl tensors by $W_{v, \varepsilon}$, 
so that

$$
\begin{array}{ll}
M_{1,1}=\left(\mathbb{C} P^{n / 2}, \tilde{g}\right), & M_{1,-1}=\left(\mathbb{C} H^{n / 2}, \tilde{g}\right), \\
M_{3,1}=\left(\mathbb{M} P^{n / 4}, \tilde{g}\right), & M_{3,-1}=\left(\mathbb{H} H^{n / 4}, \tilde{g}\right) .
\end{array}
$$

We start with a technical lemma:

Lemma 3.9. Let $\left(N^{n},\langle\cdot, \cdot\rangle\right)$ be a smooth Riemannian space locally conformally equivalent to one of the $M_{v, \varepsilon}$, so that $\tilde{g}=f\langle\cdot, \cdot\rangle$ for a positive smooth function $f=e^{2 \phi}: N^{n} \rightarrow \mathbb{R}$. Then the curvature tensor $R$ and the Weyl tensor $W$ of $\left(N^{n},\langle\cdot, \cdot\rangle\right)$ satisfy

(3-30a) $R(X, Y)=(X \wedge K Y+K X \wedge Y)+\varepsilon f(X \wedge Y+T(X, Y))$, where

$$
\begin{aligned}
T(X, Y) & =\sum_{i=1}^{v}\left(J_{i} X \wedge J_{i} Y+2\left\langle J_{i} X, Y\right\rangle J_{i}\right), \\
K & =H(\phi)-\nabla \phi \otimes \nabla \phi+\frac{1}{2}\|\nabla \phi\|^{2} \mathrm{id},
\end{aligned}
$$

(3-30b) $W(X, Y)=W_{v, \varepsilon}(X, Y)=\varepsilon f\left(-\frac{3 v}{n-1} X \wedge Y+T(X, Y)\right)$,

(3-30c) $\quad\|W\|^{2}=C_{v n} f^{2}$, where $C_{v n}=6 v n(n+2)(n-v-1)(n-1)^{-1}$,

(3-30d) $\quad\left(\nabla_{Z} W\right)(X, Y)=\varepsilon Z f\left(-\frac{3 v}{n-1} X \wedge Y+T(X, Y)\right)$

$$
\begin{aligned}
+\frac{1}{2} \varepsilon([T(X, Y), \nabla f \wedge Z]+ & T((\nabla f \wedge Z) X, Y) \\
+ & T(X,(\nabla f \wedge Z) Y)),
\end{aligned}
$$

where $X \wedge Y$ is the linear operator defined by $(X \wedge Y) Z=\langle X, Z\rangle Y-\langle Y, Z\rangle X$, $H(\phi)$ is the symmetric operator associated to the Hessian of $\phi$, and both $\nabla$ and the norm are computed with respect to $\langle\cdot, \cdot\rangle$.

Proof. The curvature tensor of $M_{\nu, \varepsilon}$ has the form

$$
\tilde{R}(X, Y)=\varepsilon\left(X \tilde{\wedge} Y+\sum_{i=1}^{\nu}\left(J_{i} X \tilde{\wedge} J_{i} Y+2 \tilde{g}\left(J_{i} X, Y\right) J_{i}\right)\right),
$$

where $(X \tilde{\wedge} Y) Z=\tilde{g}(X, Z) Y-\tilde{g}(Y, Z) X$. Under the conformal change of metric, the curvature tensor transforms as $\tilde{R}(X, Y)=R(X, Y)-(X \wedge K Y+K X \wedge Y)$. Since $\tilde{g}(X, Y)=f\langle X, Y\rangle$ and $X \tilde{\wedge} Y=f(X \wedge Y)$ and because the $J_{i}$ remain anticommuting almost Hermitian structures for $\langle\cdot, \cdot\rangle$, Equation (3-30a) follows.

The fact that the Weyl tensor has the form (3-30b) follows from the definition of $W$; the norm of $W$ can be computed directly using that the $J_{i}$ are orthogonal and that $J_{1} J_{2}= \pm J_{3}$ when $v=3$.

From

$$
\tilde{\nabla}_{Z} J_{i}=\sum_{j=1}^{\nu} \omega_{i}^{j}(Z) J_{j} \quad \text { and } \quad \tilde{\nabla}_{Z} X=\nabla_{Z} X+Z \phi X+X \phi Z-\langle X, Z\rangle \nabla \phi,
$$

where $\tilde{\nabla}$ is the Levi-Civita connection for $\tilde{g}$, we get

$$
\nabla_{Z} J_{i}=\sum_{j=1}^{v} \omega_{i}^{j}(Z) J_{j}+\left[J_{i}, \nabla \phi \wedge Z\right]
$$


(where we used the fact that $\left[J_{i}, X \wedge Y\right]=J_{i} X \wedge Y+X \wedge J_{i} Y$ ). Then

$$
\left(\nabla_{Z} T\right)(X, Y)=[T(X, Y), \nabla \phi \wedge Z]+T((\nabla \phi \wedge Z) X, Y)+T(X,(\nabla \phi \wedge Z) Y),
$$

which, together with (3-30b), proves (3-30d).

For every point $x \in M^{\prime}$, there exists a neighborhood $U$ of $x$ and a positive smooth function $f: U \rightarrow \mathbb{R}$ such that the Riemannian space $(U(x), f\langle\cdot, \cdot\rangle)$ is isometric to an open subset of one of the five model spaces $\left(M_{v, \varepsilon}\right.$ or $\left.\mathbb{R}^{n}\right)$, so at every point $x \in M^{\prime}$, the Weyl tensor $W$ of $M^{n}$ either vanishes or has the form given in (3-30b). The Jacobi operators associated to the different Weyl tensors $W_{v, \varepsilon}$ in (3-30b) differ by their multiplicities and the signs of their eigenvalues, so every point $x \in M^{\prime}$ has a neighborhood conformally equivalent to a domain of exactly one of the model spaces. Moreover, the function $f>0$ is well defined at all the points where $W \neq 0$, since $\|W\|^{2}=C_{v n} f^{2}$ by (3-30c).

By continuity, the Weyl tensor $W$ of $M^{n}$ either has the form $W_{\nu, \varepsilon}$ or vanishes at every point $x \in M^{n}$ (since $M^{\prime}$ is open and dense in $M^{n}$ - see Lemma 3.2). Moreover, every point $x \in M^{n}$ at which the Weyl tensor has the form $W_{v, \varepsilon}$ has a neighborhood in which the Weyl tensor has the same form. Hence $M^{n}=M_{0} \cup \bigcup_{\alpha} M_{\alpha}$, where $M_{0}=\{x: W(x)=0\}$ is closed, and every $M_{\alpha}$ is a nonempty open connected subset with $\partial M_{\alpha} \subset M_{0}$ such that the Weyl tensor has the same form $W_{\nu, \varepsilon}=W_{\nu(\alpha), \varepsilon(\alpha)}$ at every point $x \in M_{\alpha}$. In particular, since $M_{\alpha} \subset M^{\prime}$, each $M_{\alpha}$ is locally conformal to one of the model spaces $M_{v, \varepsilon}$.

If $M=M_{0}$ or if $M_{0}=\varnothing$, the theorem is proved. Otherwise, suppose that $M_{0} \neq \varnothing$ and that there exists at least one component $M_{\alpha}$. Let $y \in \partial M_{\alpha} \subset M_{0}$ and let $B_{\delta}(y)$ be a small geodesic ball of $M$ centered at $y$ that is strictly geodesically convex (any two points from $B(y)$ can be connected by a unique geodesic segment lying in $B_{\delta}(y)$, and that segment realizes the distance between them). Let $x \in B_{\delta / 3}(y) \cap M_{\alpha}$ and let $r=\operatorname{dist}\left(x, M_{0}\right)$. Then the geodesic ball $B=B_{r}(x)$ lies in $M_{\alpha}$ and is strictly convex. Moreover, $\partial B$ contains a point $x_{0} \in M_{0}$. Replacing $x$ by the midpoint of the segment $\left[x x_{0}\right]$ and $r$ by $r / 2$, if necessary, we can assume that all the points of $\partial B$, except for $x_{0}$, lie in $M_{\alpha}$.

The function $f$ is positive and smooth on $\bar{B} \backslash\left\{x_{0}\right\}$ (that is, on an open subset containing $\bar{B} \backslash\left\{x_{0}\right\}$, but not containing $x_{0}$ ). We are interested in the behavior of $f(x)$ when $x \in B$ approaches $x_{0}$.

Lemma 3.10. When $x \rightarrow x_{0}$ while staying in $B$, both $f$ and $\nabla f$ have a finite limit. Moreover, $\lim _{x \rightarrow x_{0}, x \in B} f(x)=0$.

Proof. The fact that $\lim _{x \rightarrow x_{0}, x \in B} f(x)=0$ follows from (3-30c) and the fact that $\left.W\right|_{x_{0}}=0$ (since $x_{0} \in M_{0}$ ). 
Since the Riemannian space $(B, f\langle\cdot, \cdot\rangle)$ is locally isometric to a rank-one symmetric space $M_{\nu, \varepsilon}$ and is also simply connected, there exists a smooth isometric immersion $\imath:(B, f\langle\cdot, \cdot\rangle) \rightarrow M_{\nu, \varepsilon}$. Since $f$ is smooth on $\bar{B} \backslash\left\{x_{0}\right\}$ and $\lim _{x \rightarrow x_{0}, x \in B} f(x)=0$, the range of $\iota$ is a bounded domain in $M_{v, \varepsilon}$. Moreover, since $\lim _{x \rightarrow x_{0}, x \in B} f(x)=0$, every sequence of points in $B$ converging to $x_{0}$ in the metric $\langle\cdot, \cdot\rangle$ is a Cauchy sequence for the metric $f\langle\cdot, \cdot\rangle$. It follows that there exists a limit $\lim _{x \rightarrow x_{0}, x \in B} l(x) \in M_{\nu, \varepsilon}$. Defining for every $x \in B$ the point $\left.\mathscr{F}\right|_{x}=\operatorname{Span}\left(J_{1}, \ldots, J_{v}\right)$ in the Grassmanian $G\left(v, \bigwedge^{2} T_{x} M^{n}\right)$, we find that there exists a limit

$$
\left.\lim _{x \rightarrow x_{0}, x \in B} \mathscr{F}\right|_{x}=:\left.\mathscr{E}\right|_{x_{0}} \in G\left(\nu, \bigwedge^{2} T_{x_{0}} M^{n}\right) .
$$

In particular, if $Z$ is a continuous vector field on $\bar{B}$, then there exists a unit continuous vector field $Y$ on $\bar{B}$ such that $Y \perp Z, \mathscr{E} Z$ on $B$. For such two vector fields, the function

$$
\theta(Y, Z)=\left\langle\sum_{j=1}^{n}\left(\nabla_{E_{j}} W\right)\left(E_{j}, Y\right) Y, Z\right\rangle
$$

(where $E_{j}$ is an orthonormal frame on $\bar{B}$ ) is well defined and continuous on $\bar{B}$. Using (3-30d), we obtain by a direct computation that at the points of $B$,

$\theta(Y, Z)=\frac{\varepsilon(n-3)}{2(n-1)}\langle(3 v \nabla f \wedge Y-(n-1) T(\nabla f, Y)) Y, Z\rangle=\frac{-3 \varepsilon v(n-3)}{2(n-1)}\langle\nabla f, Z\rangle$

(where we used that $\|Y\|=1$ and $Y \perp Z, \mathscr{g} Z$ ). Since $\theta(Y, Z)$ is continuous on $\bar{B}$, there exists a $\operatorname{limit} \lim _{x \rightarrow x_{0}, x \in B} Z f$. Since $Z$ is an arbitrary continuous vector field on $\bar{B}, \nabla f$ has a finite limit when $x \rightarrow x_{0}$ while staying in $B$.

Since $\lim _{x \rightarrow x_{0}, x \in B} f(x)=0$ and the $J_{i}$ are orthogonal, the second term on the right side of (3-30a) tends to 0 when $x \rightarrow x_{0}$ in $B$. Therefore the $(3,1)$ tensor field defined by $(X, Y) \rightarrow(X \wedge K Y+K X \wedge Y)$ has a finite limit (namely $\left.\left.R\right|_{x_{0}}\right)$ when $x \rightarrow x_{0}$ in $B$. It follows that the symmetric operator $K$ has a finite limit at $x_{0}$. Computing the trace of $K$ and using the fact that $\phi=\frac{1}{2} \ln f$, we get

$$
\triangle u=F u \text { on } B, \quad \text { where } u=f^{(n-2) / 4} \text { and } F=\frac{1}{2}(n-2) \operatorname{Tr} K .
$$

Both functions $F$ and $u$ are smooth on $\bar{B} \backslash\left\{x_{0}\right\}$ and have a finite limit at $x_{0}$. Moreover, $\lim _{x \rightarrow x_{0}, x \in B} u(x)=0$ by Lemma 3.10 and $u(x)>0$ for $x \in \bar{B} \backslash\left\{x_{0}\right\}$. The domain $B$ is a small geodesic ball, so it satisfies the inner sphere condition (the radii of curvature of the sphere $\partial B$ are uniformly bounded). By the boundary point theorem [Fraenkel 2000, Section 2.3], the inner directional derivative of $u$ at $x_{0}$ (which exists by Lemma 3.10 if we define $u\left(x_{0}\right)=0$ by continuity) is positive.

Since $\nabla u=(1 / 4)(n-2) f^{(n-6) / 4} \nabla f$ in $B$, we arrive at a contradiction with Lemma 3.10 in all cases except for $n=6$. To finish the proof in that case, we show

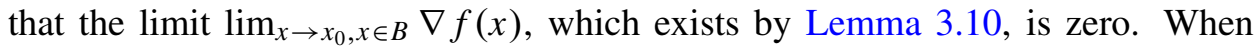
$n=6$, we have $v=1$ by (2-3), so $T(X, Y)=J X \wedge J Y+2\langle J X, Y\rangle J$, where 
$J=J(x)$ is smooth on $\bar{B} \backslash\left\{x_{0}\right\}$ and has a limit when $x \rightarrow x_{0}$ while in $B$ (see the proof of Lemma 3.10). Using the covariant derivative of $T$ computed in Lemma 3.9 and (3-30d), we obtain that on $B$,

$$
\begin{aligned}
& \left(\nabla_{U} \nabla_{Z} W\right)(X, Y) \\
& =\varepsilon\langle H(f) U, Z\rangle\left(-\frac{3}{5} X \wedge Y+T(X, Y)\right) \\
& +\frac{1}{2} \varepsilon([T(X, Y), H(f) U \wedge Z]+T((H(f) U \wedge Z) X, Y)+T(X,(H(f) U \wedge Z) Y)) \\
& +\frac{1}{2} \varepsilon f^{-1} Z f([T(X, Y), \nabla f \wedge U]+T((\nabla f \wedge U) X, Y)+T(X,(\nabla f \wedge U) Y)) \\
& +\frac{1}{4} \varepsilon f^{-1}[[T(X, Y), \nabla f \wedge U]+T((\nabla f \wedge U) X, Y) \\
& +T(X,(\nabla f \wedge U) Y), \nabla f \wedge Z] \\
& +\frac{1}{4} \varepsilon f^{-1}([T((\nabla f \wedge Z) X, Y), \nabla f \wedge U]+T((\nabla f \wedge U)(\nabla f \wedge Z) X, Y) \\
& +\frac{1}{4} \varepsilon f^{-1}([T(X,(\nabla f \wedge Z) Y), \nabla f \wedge U]+T((\nabla f \wedge U) X,(\nabla f \wedge Z) Y) \\
& \quad+T(X,(\nabla f \wedge U)(\nabla f \wedge Z) Y)),
\end{aligned}
$$

where $H(f)$ is the symmetric operator associated to the Hessian of $f$. Taking $U=Z=E_{j}$, where $\left\{E_{j}\right\}$ is an orthonormal basis, and summing up by $j$ we find after some computation

$$
\begin{aligned}
\sum_{j=1}^{6}\left(\nabla_{E_{j}} \nabla_{E_{j}} W\right)(X, Y) & \\
=\varepsilon \triangle & f\left(-\frac{3}{5} X \wedge Y+T(X, Y)\right)-\varepsilon f^{-1}\|\nabla f\|^{2} T(X, Y) \\
& +\varepsilon f^{-1}(T(X, Y) \nabla f \wedge \nabla f+T((X \wedge Y) \nabla f, \nabla f)) \\
& \quad+\frac{3}{2} \varepsilon f^{-1}(\nabla f \wedge(X \wedge Y) \nabla f+J \nabla f \wedge(X \wedge Y) J \nabla f) .
\end{aligned}
$$

Since both $\nabla f$ and $J$ are smooth on $\bar{B} \backslash\left\{x_{0}\right\}$ and have limits when $x \rightarrow x_{0}$ while in $B$, there exist unit vector fields $X$ and $Y$ that are continuous on $\bar{B}$ and satisfy

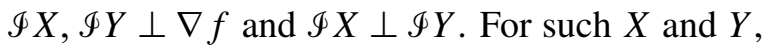

$$
\sum_{j=1}^{6}\left(\nabla_{E_{j}} \nabla_{E_{j}} W\right)(X, Y)=\varepsilon \triangle f\left(-\frac{3}{5} X \wedge Y+J X \wedge J Y\right)-\varepsilon f^{-1}\|\nabla f\|^{2} J X \wedge J Y .
$$

Since the left side is continuous on $\bar{B}$ and $\lim _{x \rightarrow x_{0}, x \in B} \triangle f=0$ by (3-31) and Lemma 3.10, we obtain that the field $f^{-1}\|\nabla f\|^{2} J X \wedge J Y$ of skew-symmetric operators has a limit at $x_{0}$. Taking the trace of its square, we find that there exists a limit $\lim _{x \rightarrow x_{0}, x \in B} f^{-2}\|\nabla f\|^{4}$, which implies $\lim _{x \rightarrow x_{0}, x \in B} \nabla f=0$ by Lemma 3.10. We again arrive at a contradiction with the boundary point theorem for the function $u=f$ satisfying (3-31). 


\section{References}

[Atiyah et al. 1964] M. F. Atiyah, R. Bott, and A. Shapiro, "Clifford modules", Topology 3: suppl. 1 (1964), 3-38. MR 29 \#5250 Zbl 0146.19001

[Blažić and Gilkey 2004] N. Blažić and P. Gilkey, "Conformally Osserman manifolds and conformally complex space forms", Int. J. Geom. Methods Mod. Phys. 1 (2004), 97-106. MR 2005c:53034 Zbl 1076.53038

[Blažić and Gilkey 2005] N. Blažić and P. Gilkey, "Conformally Osserman manifolds and selfduality in Riemannian geometry", pp. 15-18 in Differential geometry and its applications, edited by J. Bureš et al., Matfyzpress, Prague, 2005. MR 2007k:53032 Zbl 1117.53028

[Blažić et al. 2005] N. Blažić, P. Gilkey, S. Nikčević, and U. Simon, "The spectral geometry of the Weyl conformal tensor", pp. 195-203 in PDEs, submanifolds and affine differential geometry, edited by B. Opozda et al., Banach Center Publications 69, Polish Acad. Sci., Warsaw, 2005. MR 2006k:53044 Zbl 1091.53007

[Blažić et al. 2008] N. Blažić, P. Gilkey, S. Nikčević, and I. Stavrov, "Curvature structure of selfdual 4-manifolds", Int. J. Geom. Methods Mod. Phys. 5:7 (2008), 1191-1204. MR 2010a:53148 Zbl 1163.53010

[Chi 1988] Q.-S. Chi, "A curvature characterization of certain locally rank-one symmetric spaces", J. Differential Geom. 28:2 (1988), 187-202. MR 90a:53060 Zbl 0654.53053

[Derdziński and Shen 1983] A. Derdziński and C. L. Shen, "Codazzi tensor fields, curvature and Pontryagin forms”, Proc. London Math. Soc. (3) 47 (1983), 15-26. MR 84h:53048 Zbl 0519.53015

[Fraenkel 2000] L. E. Fraenkel, An introduction to maximum principles and symmetry in elliptic problems, Cambridge Tracts in Math. 128, Cambridge University Press, 2000. MR 2001c:35042 Zbl 0947.35002

[García-Río et al. 2002] E. García-Río, D. N. Kupeli, and R. Vázquez-Lorenzo, Osserman manifolds in semi-Riemannian geometry, Lecture Notes in Mathematics 1777, Springer, Berlin, 2002. MR 2003e:53052 Zbl 1005.53040

[Gilkey 2001] P. B. Gilkey, Geometric properties of natural operators defined by the Riemann curvature tensor, World Scientific, River Edge, NJ, 2001. MR 2002k:53052 Zbl 1007.53001

[Gilkey 2007] P. B. Gilkey, The geometry of curvature homogeneous pseudo-Riemannian manifolds, ICP Advanced Texts in Mathematics 2, Imperial College Press, London, 2007. MR 2008k:53151 Zbl 1128.53041

[Gilkey et al. 1995] P. Gilkey, A. Swann, and L. Vanhecke, "Isoparametric geodesic spheres and a conjecture of Osserman concerning the Jacobi operator", Quart. J. Math. Oxford Ser. (2) 46:183 (1995), 299-320. MR 96h:53051 Zbl 0848.53023

[Harvey and Lawson 1982] R. Harvey and H. B. Lawson, Jr., "Calibrated geometries", Acta Math. 148 (1982), 47-157. MR 85i:53058 Zbl 0584.53021

[Husemoller 1975] D. Husemoller, Fibre bundles, 2nd ed., Graduate Texts in Mathematics 20, Springer, New York, 1975. MR 51 \#6805 Zbl 0307.55015

[Lawson and Michelsohn 1989] H. B. Lawson, Jr. and M.-L. Michelsohn, Spin geometry, Princeton Mathematical Series 38, Princeton University Press, 1989. MR 91g:53001 Zbl 0688.57001

[N 2003] Y. Nikolayevsky, "Osserman manifolds and Clifford structures", Houston J. Math. 29:1 (2003), 59-75. MR 2003k:53048 Zbl 1069.53042

[N 2004] Y. Nikolayevsky, “Osserman manifolds of dimension 8”, Manuscripta Math. 115:1 (2004), 31-53. MR 2005m:53049 Zbl 1065.53034 
[N 2005] Y. Nikolayevsky, "Osserman conjecture in dimension $n \neq 8,16$ ", Math. Ann. 331:3 (2005), 505-522. MR 2005k:53038 Zbl 1075.53016

[N 2006] Y. Nikolayevsky, "On Osserman manifolds of dimension 16”, pp. 379 - 398 in Contemporary Geometry and Related Topics (Belgrade, 2005), edited by N. Bokan et al., Faculty of Mathematics, University of Belgrade, 2006.

[Olszak 1989] Z. Olszak, "On the existence of generalized complex space forms", Israel J. Math. 65:2 (1989), 214-218. MR 90c:53091 Zbl 0674.53061

[Osserman 1990] R. Osserman, "Curvature in the eighties", Amer. Math. Monthly 97:8 (1990), 731756. MR 91i:53001 Zbl 0722.53001

[Pfister 1995] A. Pfister, Quadratic forms with applications to algebraic geometry and topology, London Math. Soc. Lecture Note Ser. 217, Cambridge University Press, 1995. MR 97c:11046 Zbl 0847.11014

Received January 31, 2009.

YURI NIKOLAYEVSKY

DEPARTMENT OF MATHEMATICS AND STATISTICS

LA TROBE UNIVERSITY

Melbourne, Victoria 3086

Australia

y.nikolayevsky@latrobe.edu.au 